This item was submitted to Loughborough's Research Repository by the author.

Items in Figshare are protected by copyright, with all rights reserved, unless otherwise indicated.

\title{
Measurements of fuel thickness for prefilming atomisers at elevated pressure
}

PLEASE CITE THE PUBLISHED VERSION

https://doi.org/10.1016/j.jimultiphaseflow.2020.103313

PUBLISHER

Elsevier

VERSION

AM (Accepted Manuscript)

PUBLISHER STATEMENT

This paper was accepted for publication in the journal International Journal of Multiphase Flow and the definitive published version is available at https://doi.org/10.1016/j.ijmultiphaseflow.2020.103313

\section{LICENCE}

CC BY-NC-ND 4.0

\section{REPOSITORY RECORD}

Brend, Mark, Ashley Barker, and Jon Carrotte. 2020. "Measurements of Fuel Thickness for Prefilming Atomisers at Elevated Pressure". Loughborough University. https://hdl.handle.net/2134/13097732.v1. 


\title{
Measurements of fuel thickness for prefilming atomisers at elevated pressure
}

\author{
M. A. Brend ${ }^{\mathrm{a}, *}$, A. G. Barker ${ }^{\mathrm{a}}$, J. F. Carrotte ${ }^{\mathrm{a}}$ \\ ${ }^{a}$ National Centre for Combustion and Aerothermal Technology (NCCAT), Loughborough University, Loughborough, LE11 3TU, UK
}

\begin{abstract}
This work describes an experimental study of the fuel flows on the prefilmer of an aerospace gas turbine airblast atomiser at elevated pressure. The work identifies the physics leading to contradictory findings within the literature. This concerns an important atomisation boundary condition, whether the thickness of the fuel film on the prefilming surface influences the downstream drop size distribution. Analysis of the experimental data shows that fuel film thickness becomes uncorrelated with the downstream drop size if surface tension forces dominate inertia at the prefilmer tip. Fuel film thickness however provides the initial length scale for primary atomization if the fuel inertia forces exceed surface tension. It is the high inertial conditions that are associated with gas turbine operation, but the low inertial conditions that are readily achievable at laboratory scale through momentum flux scaling. Additionally, a detailed statistical description of the fuel flow has been provided for the atomiser tested. This reveals the importance of upstream hydrodynamic and aerodynamic boundary conditions on the probability of a ligament forming. Surprisingly, operating pressure is shown to have limited effect on the probability of ligament formation, a significant advantage for future modelling of the primary atomization processes.
\end{abstract}

Keywords: ERLIF, liquid film thickness, atomisation, fuel preparation, gas turbine combustion, elevated pressure

\section{Introduction}

The future growth of aero gas turbine products is dependent on the ability to deliver propulsion systems that reduce the environmental impact of aviation. For example, the European Vi5 sion 2020/Flight 2050 targets $75 \%$ and $90 \%$ reductions in $\mathrm{CO}_{2}$ and $N O_{x}$ respectively (Darecki et al., 2011). To achieve these objectives requires improvements in existing rich burn combustion system emissions performance together with the develop- ${ }^{35}$ ment of new lean burn technology. A key aspect of reducing emissions in these types of combustion system architectures is the ability to deliver high quality atomisation of the liquid fuel. This must be achieved across the complete engine operating range.

A variety of atomiser designs have been investigated for use within gas turbines. These include liquid jet and sheet atomisers for which investigations have been undertaken to identify the dominant instability break-up mechanisms (Wu et al., 1997; Bremond et al., 2007; Lee et al., 2007; Fuster et al., 2013). ${ }^{45}$ In aerospace applications however, atomisation is more typically achieved using pre-filming air-blast atomisers. In such configurations liquid fuel passes through several internal fuel passages, within the injector, prior to being introduced onto a prefilmer where a liquid film is formed. Upstream of this pre- ${ }^{50}$ filmer, vanes are used to impart swirl to an air stream which subsequently passes over the liquid film (Barker \& Carrotte, 2014). As the film reaches the end of the prefilmer, a second, swirling air stream is introduced outboard. This is arranged so

\footnotetext{
${ }^{*}$ Corresponding author

Email address: m. brend@lboro.ac.uk (M. A. Brend)
}

that the liquid break up and atomisation occurs between these two air streams. To understand the resultant break-up process is challenging since it is associated with complex aerodynamic flow field features, two phase flows and various types of breakup phenomenon. Furthermore, it is an extremely difficult region to interrogate experimentally whilst the nature of the problem makes it numerically challenging.

Some of the key operating parameters that affect the breakup process have already been identified in the literature. This has typically been achieved using non-contact optical measurement techniques which characterise the spray in the injector far field. Work undertaken by several authors has demonstrated the importance of air velocity (e.g. Lefebvre (1980); Jasuja (1981)) and air pressure (e.g. Lefebvre (1980); Jasuja (1981); El-Shanawany \& Lefebvre (1980); Bhayaraju \& Hassa (2006)) on droplet size. Essentially increases in these quantities result in increased air momentum, and hence increased aerodynamic forces, relative to the liquid surface tension forces which resist the liquid break-up process. Unsurprisingly therefore, liquids with higher surface tension result in increased droplet sizes whilst, for aero engine applications, liquid viscosity has been shown to have only a small effect (Lefebvre, 1980). However, one area of confusion concerns the effect of liquid film thickness. For example, some workers (Rizk \& Lefebvre, 1980; ElShanawany \& Lefebvre, 1980; Déjean et al., 2016) have shown that an increase in liquid film thickness results in an increase in drop size. However, other workers (Sattelmayer \& Wittig, 1986; Aigner \& Wittig, 1988; Gepperth et al., 2013; Shanmugadas et al., 2018) have indicated no such effect. For these cases more detailed investigations have suggested that liquid accumulates at the edge or tip of the atomiser. When this accumu- 
lation has reached a critical depth, primary atomisation events ench that the droplet size is controlled by the thickness of the critical depth rather than the initial film thickness. Based on these observations phenomenological models describing the liquid phase have been developed with emphasis on the film 120 and its atomisation at the prefilmer edge (Chaussonnet et al.,

65 2013; Warncke et al., 2017). These models have also been incorporated into large eddy simulations (LES) (Warncke et al., 2017).

The application of techniques such as shadowgraphy to pro-125 vide qualitative information on the break-up process has be-

70 come commonplace. The use of suitable image processing methods has then allowed quantitative data on ligament breakup lengths, downstream of the prefilmer edge to be obtained (Carvalho \& Heitor, 1998). To provide better optical access ${ }_{130}$ many investigations use simplified 2-dimensional 'planar' rep-

75 resentations of axisymmetric fuel injectors (e.g. Gepperth et al. (2012)). Fuel velocity estimates can also be obtained by observing the displacement of the liquid phase between 2 instances in time (Bhayaraju \& Hassa, 2006). Various means of measuring ${ }_{135}$ film thickness have also been employed and applied to planar

80 fuel injector models. These tend to be point based and examples include a needle contact technique (Rizk \& Lefebvre, 1980) and a laser focus displacement meter (Gepperth et al., 2010). Measurements of this type have provided valuable insight into ${ }_{140}$ the break-up mechanisms associated with this style of atomiser.

85 However, the use of a simplified 2-dimensional representation of the atomiser does have limitations. For example, none of the aerodynamic flow field features present within a representative injector, such as vanes wakes and swirling flows, can be ${ }_{145}$ included.

90 In contrast to many previous investigations, the measurements presented here have been undertaken on a prefilming air-blast atomiser typical of that proposed for future, low emission, gas turbine engines. The aerodynamic flow across the pre-150 filmer is therefore fully featured, containing aerodynamic structures that would be present within the engine environment. The fuel is introduced onto the conical prefilmer via fuel slots and contains a tangential component about the injector centreline. Hence, it is to be anticipated that both the fuel and airflow ap-155 proach the end of the prefilmer at an oblique angle. The injector flow field then issues into a downstream geometry in which the boundary conditions are broadly representative of those found within an engine environment at subcritical conditions. The flow is radially confined, to simulate the presence of flame tube ${ }_{160}$ liners (Fu et al., 2005), whilst adjacent injectors are also present to simulate the aerodynamic boundary conditions that are typically associated with annular combustors (Vespermann, 2018). To undertake measurements in this environment is challenging and optical access significantly restricted.

To overcome these challenges, this article describes the de-

110 velopment of an experimental technique to measure the thickness of fuel films using a line of sight ratiometric fluorescence technique. This is based upon the work of Hidrovo and Hart (Hidrovo \& Hart, 2001) and uses emission reabsorption ${ }_{170}$ laser induced fluorescence (ERLIF) to eliminate excitation non- filmer surface. This has enabled high fidelity measurements of spatially distributed film thickness and ligament statistics to be obtained over a range of operating pressures. Because the measurements require only a single line of sight to the measurement region, they may be performed using a single point of access for illumination and imaging. Previously suggested noncontact methods for quantitative thickness measurement were considered (Reddyhoff et al., 2008; Gepperth et al., 2010; Feldman et al., 2018), but thought impractical due the access challenges, and issues associated with total internal reflection Häber et al. (2015). Thickness measurement based upon the brightness based laser induced fluorescence method was also considered (Alekseenko et al., 2015). For measurements in dense spray on a complex geometry however, control of illumination uniformity due to localised directional scatter from geometry and attenuation arising from the spray itself can give rise to significant uncertainty. The ERLIF method, however, was developed specifically to eliminate errors of this form and, given the single line of sight access requirement, is ideally suited to complex measurements in representative environments.

By performing these measurements on the same lean direct injection (LDI) prefilming style air-blast atomiser tested by Barker \& Carrotte (2014), the structures measured on the prefilmer in this work may be associated with the downstream spray distribution previously published. Data showing mean drop diameter and fuel mass flux collected using phase Doppler anemometry (PDA) at momentum flux scaled, atmospheric pressure conditions have therefore been reproduced with permission and are shown in Figure 1. The measured fuel volume flux, shown in Figure 1b exhibits clear peaks located on two concentric circular arcs. The plots have been annotated, to denote the feature within the atomiser that has resulted in the formation of localised peaks.

The total number of peaks observed on the inner arc was found to correspond to the number of gas phase turning vanes located within the inner passage. The inner passage swirler has 18 vanes, the wakes of which pass across the prefilmer surface. Hence, for a quadrant measurement, 4.5 vanes would be expected to be observed. As the measurement was performed some distance downstream of the atomiser in order to permit beam access, some movement of the measured wakes is routinely observed, as is suggested by the pairing of the markers added to the inner arc. It is therefore reasonable to expect 4 wake structures to be measured in one sector and 5 in the adjacent. Applying a similar analysis to the outer arc features, 6 wakes are found, corresponding to the 22 outer passage turning vanes. This emphasises the importance of the detailed gas flow across the prefilmer in providing boundary conditions for atomisation.

Of the wakes observed on the inner arc, two have particularly high mass flux, and correspond to elevated levels of mean drop diameter. These structures were associated with a fuel port located in the internal fuel galleries, which was previously identified by flow visualisation (Barker \& Carrotte, 2014). The device tested was fitted with eight discrete fuel ports and so two peaks are observed in the quadrant measurement. These in turn have a significant affect on the drop diameter shown in Figure 1a. 


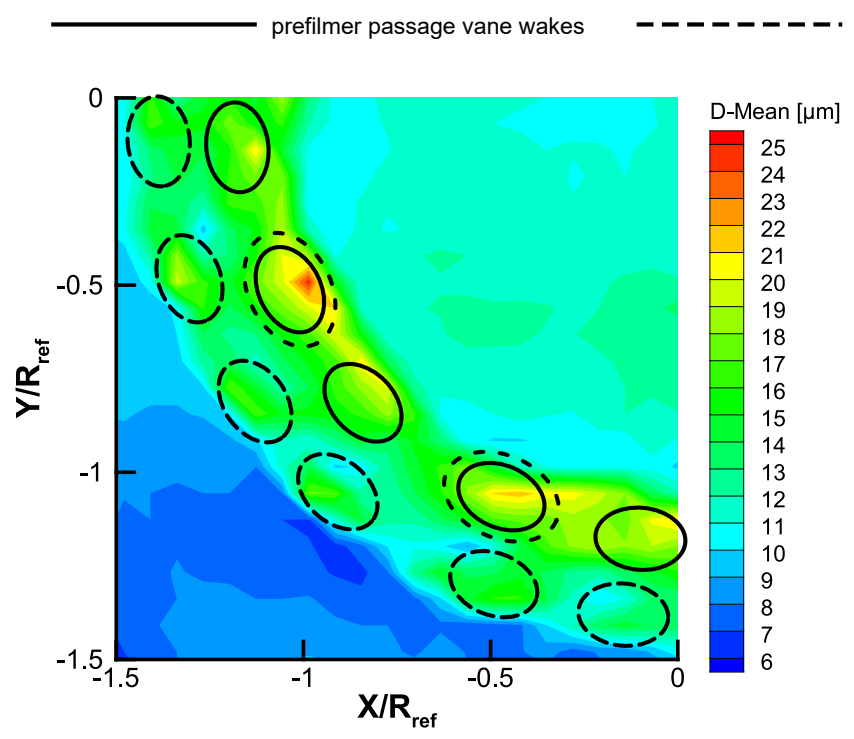

(a) mean drop diameter outer passage vane wakes
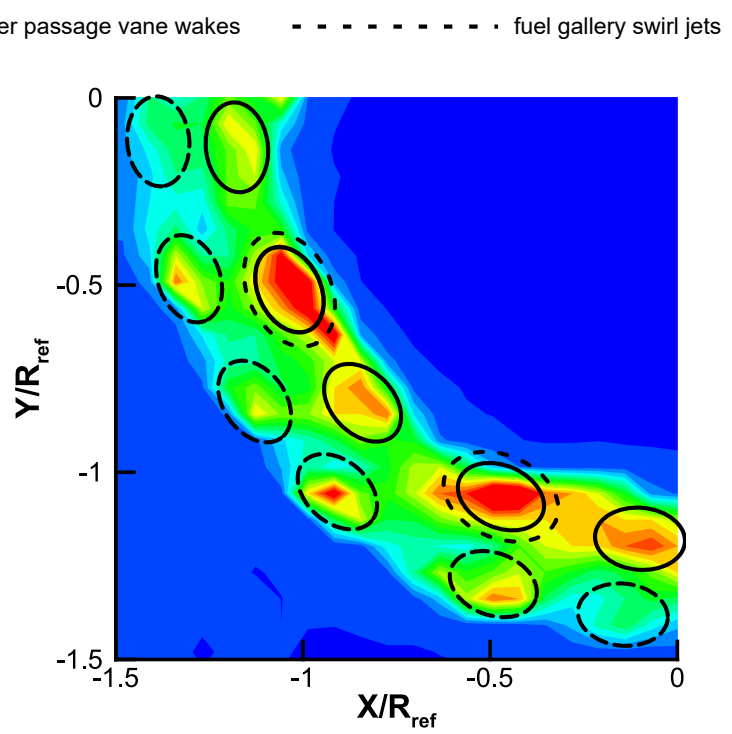

(b) fuel volume flux

Figure 1: Fuel measurements performed downstream of an engine representative atomiser. PDA data has been acquired by area scanning across a quadrant of the atomiser efflux. Local peaks in the spatial distribution corresponding to upstream aerodynamic and fuel delivery features have been highlighted. Reproduced from Barker \& Carrotte (2014) with permission, (c) Copyright Rolls-Royce plc 2014.

Identically placed markers have been added to the mean drop diameter plot shown in Figure 1a to indicate the correspondence of fuel mass flux with drop size distribution, the flux being derived from both drop size and velocity. There is correspondence 205 in structure and phasing between the fuel volume flux measurement and droplet diameter, although some differences are observed. The peaks measured in the mean drop diameter field do not correspond exactly to those within the fuel mass flux, indicating mismatch between the droplet velocity field and the 210 droplet diameter.

This paper makes use of the ERLIF film thickness measure- ${ }^{21}$ ment system developed to determine the detailed behaviour of the fuel on the prefilming surface of an LDI air-blast atomiser at elevated pressure conditions. Instantaneous and average thickness fields are used to describe the transport processes occurring on the prefilming surface as well as the physical mechanisms which deliver fuel to the prefilmer tip and into the priatomisation zone. Quantitative comparisons of thickness between the momentum flux scaled test cases are performed $\mathrm{d}_{220}$ by calculation of bulk thicknesses, with the measured thickness averaged circumferentially and in time. This data has additionally used to define the bulk fuel velocities on the prefilmer surface, allowing the relative significance of fuel inertia and surface tension to be assessed at the tip. This is then shown to be 225 significant in determining primary atomisation behaviour. ERLIF measurements are additionally used to generate probability density functions of ligament length and ligament circumferential position in order to quantitatively assess the impact of pressure condition and prefilmer boundary conditions on primary 230 $_{20}$ atomisation behaviour.

\section{Experimental Methods}

\subsection{Test facility}

Fuel thickness measurements have been performed on the elevated pressure spray test facility at Loughborough University. A schematic detailing the air/fuel supply and control systems is shown in Figure 3. Up to $1 \mathrm{~kg} / \mathrm{s}$ of air at 8 bar may be supplied via a safety shut-off valve. Mass flow rate metering is performed using an Emerson Coriolis meter and the test condition set using two Schubert and Salzer gate valves. Test section inlet pressure is restricted to a maximum of 4.5 bara by a safety relief valve located at the test cell delivery point. Static pressure at atomiser inlet and the pressure drop across the installed atomisers was monitored using absolute and differential transducers respectively.

Figure 2: FIGURE REMOVED ON REVIEW

To provide representation of annular combustor aerodynamics within the test section, air was fed through three airblast atomisers. These were mounted such that they exhausted into a liner with dimensions nominally equivalent to an aerospace combustion chamber. The liner was then mounted into the pressure vessel, permitting operation and conditions exceeding ambient. To minimise the disruption of the optical viewing condition, fuel was only supplied to the central atomiser being studied. The adjacent atomisers were used only for the provision of representative aerodynamic boundary conditions to the central atomiser. To perform the film thickness measurements an ERLIF instrument was developed (details of which are provided in section 2.3), requiring a single unobstructed view of the atomiser prefilming surface. In order to gain this optical access, a viewing tube was used to penetrate the liquid spray and fitted 


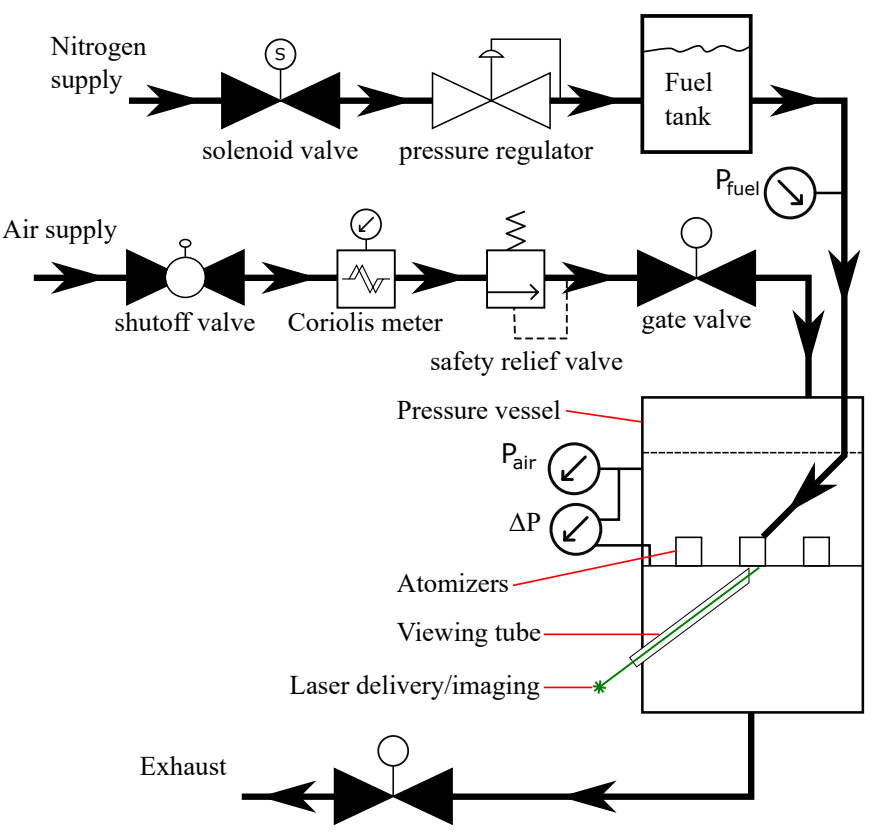

Figure 3: A schematic illustrating the air/fuel process control system.

to the side wall of the test section. This was precisely oriented to provide a view orthogonal to the prefilming surface.

To ensure the air flow swirl angles and fuel distribution on the prefilmer closely match those within a real aerospace application, a modified prototype atomiser targeting future LDI applications was tested. The atomiser matches that previously 270 studied by Barker \& Carrotte (2014), who performed a full survey of the velocity, turbulence and pressure fields inside the atomiser gas flow passages as well as measuring the drop size distribution downstream of the secondary atomisation region. A schematic illustrating the atomiser configuration is shown in 275 Figure 4. The inner most air passages, which correspond to a rich pilot module have been omitted. The pilot module was not fueled, but provided representative recirculation zones, vortex precession and turbulence in the downstream region. The prefilmer surface is located between two passages (here named pre-280 filmer passage and outer passage) which aim to deliver high levels of shear at the tip of the prefilmer along with swirl in order to stabilise the flame under reacting conditions. For the design considered here, the swirl direction of the airflow within the prefilmer and outer passages is the same, but the level of $\operatorname{swirl}_{285}$ imparted differs. Similarly, the swirl direction of the fuel stream corresponds to that of the air stream, but the level of swirl imparted also differs. The outer diameter of the atomiser and so the issuing air jet is $O(100 \mathrm{~mm})$, the prefilmer length $O(10 \mathrm{~mm})$ and both the prefilmer step and tip $O(1 \mathrm{~mm})$.

In order to deliver fuel to the central atomiser, a nitrogen pressurised supply with a gas bottle regulator was used to pressurise a fuel reservoir. This was filled with Exxsol D80, which had been doped with low concentrations of fluorescent dye. The reservoir outlet was connected to the central atomiser with fuel 295 pressure measured using an absolute pressure transducer. Offline testing was performed to determine the fuel system flow-

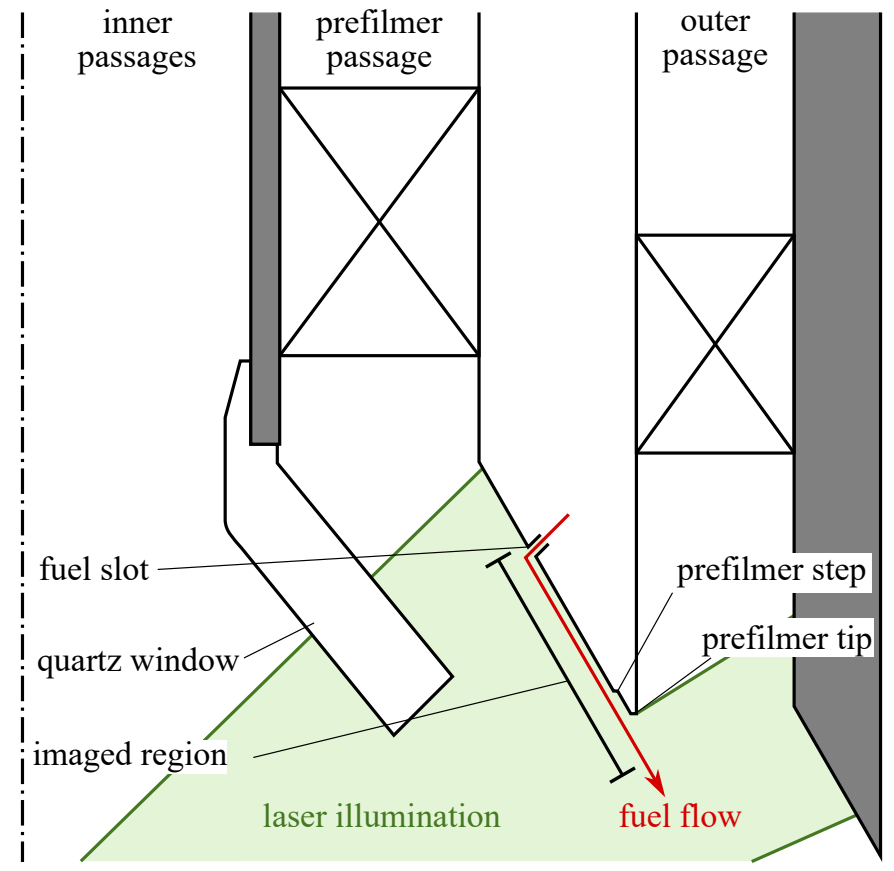

Figure 4: A schematic illustrating the geometry of the tested atomiser.

pressure drop measurements.

Fuel is delivered onto the prefilmer through a series of injector passages and galleries to a fuel slot. These passages contain a set of eight angled ports in order to form fuel jets in order to pre-swirl the fuel prior to delivery onto the prefilmer surface from where it is transported towards the tip. Before reaching the prefilmer tip, the fuel crosses a backwards facing step whose size approximates that of the tip. The ERLIF instrument and associated viewing tube was arranged in order to give a full view of this fuel flow and initial primary atomisation. The imaged region and laser illumination are shown in Figure 4.

The prototype atomiser received minor modification to permit imaging across the full length of the prefilmer surface. A conical metal feature separating the inner passage airflow from the prefilmer passage airflow has been replaced with a similar component manufactured from quartz. In order that this be reliably manufactured and bonded to the prototype, the quartz window was manufactured thicker than the replaced metal component. To minimise disruption of the prefilmer flows, this additional thickness has been added to the inner passage side of the window, i.e. the prefilmer passage geometry remains unchanged. As the mean radius of the passage increases, the passage heigh decreases. The air flow trajectory along the prefilmer length is therefore modified from that imparted by the turning vanes. Similarly the fuel flow enters the passages and galleries upstream of the slot with some swirl and undergoes both area and radial coordinate changes. It therefore is also modified from the upstream swirl imparted by the geometry. Because the angles of the vanes and upstream swirl jets are known from design data, an estimate of the relative swirl of the three streams may be made, even if the absolute swirl numbers at the prefilmer surface are not known: the fuel stream has the lowest swirl angle; then the prefilmer passage air flow; the outer pas- 
sage air flow the highest swirl angle. Were fuel shed from the ar to move radially outboard, it would cross the outer air passage. Because of the difference in trajectory, this would $\mathrm{d}_{350}$ result in significant aerodynamic forcing, effectively forming a jet in cross flow type condition.

\subsection{Operating conditions}

All tests were performed at isothermal conditions, with fixed ${ }^{355}$ relative pressure drop across the atomiser, thus maintaining constant Mach number. Fuel flow rates have been momentum flux scaled from an appropriate cruise condition. Test parameters and derived non-dimensional numbers are summarised in Table 1. Each operating condition tested is referred to by ${ }^{360}$ the upstream static pressure set point, which is expressed in absolute barometric units (bara). The film loading is calculated as the liquid volume flux per unit breadth, which, given the conical nature of the prefilmer, varies along its length. The relative pressure drop $\left(\Delta P_{a} / P\right)$ and momentum flux ratio are ${ }^{36}$ calculated from instrumentation measurements. For the tests performed, the range of relative pressure drop was measured to be $\pm 5 \%$ of the set-point value which was selected to deliver engine representative Mach numbers across the prefilmer. The range of fuel mass flow rates, relative to the set point was how-370 ever somewhat larger, $\pm 15 \%$ of the set point value, with variations observed between configuring the fuel flow and performing the fluorescence measurement. Air flow Reynolds numbers are high throughout, indicating small direct viscous effects.

Reynolds number was calculated from air density $\rho_{a}$, dynamic viscosity $\mu_{a}$, characteristic velocity $U_{a}$ and length $L_{a}$; $R e=\rho_{a} U_{a} L_{a} / \mu_{a}$. Here the characteristic velocity is defined in terms of the fuel injector pressure drop $U_{a}=\sqrt{2 \Delta P_{a} / \rho_{a}}$ and the length scale is based on the outer diameter of the fuel injector jet flow at point of exhaust. The viscosity and density were calculated from the gas temperature at inlet to the facility, $281 \mathrm{~K}$ and gas static pressure. For the operating conditions tested $U_{a}$ is $O(100 \mathrm{~m} / \mathrm{s})$, with all velocities at high incompressible speeds. Similarly the Weber number is calculated from the aerodynamic force specified by $\rho_{a}$ and $U_{a}$, the scale of the fuel droplets $L_{f}$ and the surface tension $\sigma_{f}(1)$.

$$
W e=\rho_{a} U_{a}^{2} L_{f} / \sigma_{f}
$$

Here $L_{f}$ was chosen based upon the physical geometry of the atomiser and is $O(1 \mathrm{~mm})$. The momentum flux ratio $q$ was calculated from the prefilmer passage pressure loss coefficient ${ }_{37}$ $\lambda$ and flow number $F_{N}$ for the fuel circuit, the relation is shown in (2), where $A_{f}$ is the sectional area of the fuel slot annotated in Figure 4. For the experiments described here, the fuel used was Exxsol D80. This is a Newtonian fluid with density $\rho_{f}=796 \mathrm{kgm}^{-3}$, dynamic viscosity $\mu_{f}=1.66 \mathrm{mPas}$ and surface tension $\sigma_{f}=26.3 \mathrm{mNm}^{-1}$. By controlling the pressure drop across the atomiser $\left(\Delta P_{a}\right)$, momentum flux ratio was held constant for each of the tests performed, $q=90$.

$$
q=\frac{\rho_{a} U_{a}^{2}}{\rho_{f} U_{f}^{2}}=\frac{2 \Delta P_{a}}{\lambda} \frac{A_{f}^{2}}{F_{N} \Delta P_{f}}
$$

\subsection{Measurement technique}

Measurement of the thickness of the fuel film on the prefilmer was performed using the ERLIF technique. By simply doping the fuel delivered to the atomiser with two appropriately chosen fluorescent dyes, the thickness of the fuel film may be measured from the ratio of the fluorescent return. The implementation closely follows the design of Hidrovo and Hart Hidrovo \& Hart (2001), where full details of the technique may be found. For successful implementation of ERLIF, it is important that the dyes chosen have emission spectra with minimal overlap, in order that an image of each dye be captured independently, with minimal cross-talk. Additionally, it is required that the light emitted by the shorter wavelength dye be reabsorbed by the longer wavelength dye. This reabsorption leads to the disparity in fluorescent return and results in the linearity property that enables recovery of thickness from the ratio of fluorescent return. For good performance of the technique, ideally the two laser dyes should provide similar light return from the chosen excitation source. A high absorption coefficient of the longer wavelength dye is highly desirable as this maximises the reabsorption of the shorter wavelength dye. For the study discussed here, Pyromethene 567, Pyromethene 650 dyes each mixed with Exssol D80 at a concentration of $3 \times 10^{-4} \mathrm{~mol} / \mathrm{litre}$ were used, following Hidrovo et al. (2004).

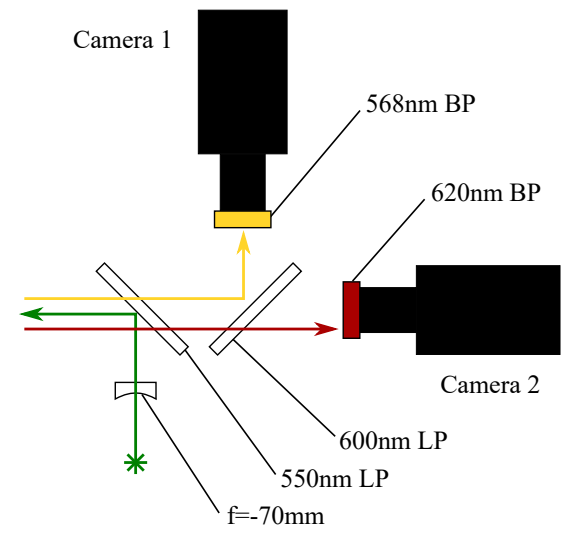

Figure 5: A schematic illustrating the optical arrangement used for ERLIF measurement.

A custom optical instrument was designed and manufactured by Etalon Research Ltd in order to combine the views of two cameras, illustrated schematically in Figure 5. Excitation of the Pyromethene dyes was performed using a frequency doubled, Q-Switched Nd:YAG laser (Litron Nano-TPIV 135-15), this was simply beam expanded using an $\mathrm{f}=-$ $70 \mathrm{~mm}$ plano convex singlet with an appropriate coating. The

Table 1: Operating conditions

\begin{tabular}{lcccc}
\hline Setpoint (bara) & $\mathbf{1 . 5}$ & $\mathbf{2 . 0}$ & $\mathbf{3 . 0}$ & $\mathbf{3 . 5}$ \\
\hline Inlet air pressure (bara) & 1.53 & 2.02 & 2.98 & 3.52 \\
Film loading, fuel slot $\left(\mathbf{c m}^{2} / \mathbf{s}\right)$ & 0.513 & 0.566 & 0.755 & 1.01 \\
Film loading, prefilmer tip $\left(\mathbf{c m}^{2} / \boldsymbol{s}\right)$ & 0.451 & 0.498 & 0.663 & 0.887 \\
Air Reynolds number & $470 \mathrm{k}$ & $630 \mathrm{k}$ & $940 \mathrm{k}$ & $1150 \mathrm{k}$ \\
Weber number & 157 & 213 & 317 & 403 \\
\hline
\end{tabular}


laser head was equipped with a variable half wave plate prior ation of the emitted laser beams. Two Etalon Research RTCam4 cameras, fitted with Nikon 200mm telephoto lenses and hard coated bandpass filters were used for imaging. The bandpass filters were selected to match the peak emission bands $620 \mathrm{~nm}$, 10nm FWHM, OD4 attenuation). The camera views were combined using a $90 \times 75 \mathrm{~mm}, 600 \mathrm{~nm}$ longpass dichroic mirror, resulting in a mirror of the image recorded by camera 1. A second $90 \times 75 \mathrm{~mm}$ dichroic mirror (550nm longpass) was

390 then used to coalign the expanded beam from the laser with the camera views. The entire instrument was rigidly assembled, such that it could be positioned in any orientation. This implementation permitted acquisition frequencies up to $15 \mathrm{~Hz}$ and a spatial resolution of $18 \mu \mathrm{m} / \mathrm{px}$, although both could be increased using high repetition rate cameras and lasers or higher resolution imaging configurations.

\subsection{Calibration}

After the cameras were focussed onto the prefilmer surface, ${ }_{435}$ the instrument was calibrated in situ. A geometric calibration wasing a LaVision Type 7 target and the DaVis 8.2 image analysis software to ensure good registration of the two camera views. This acts to remove the mirroring introduced into one of the camera views through dichroic separa- ${ }_{440}$ tion of the two fluorescent emissions. Additionally the geomet405 ric calibration is used to eliminate any residual displacement arising from small differences in the imaging systems. Subsequent thickness calibration was performed to relate the measured ERLIF ratio to a known thickness $\hat{t}$. This thickness has been non-dimensionalised by the length scale $L_{f}$, giving a nondimensional thickness of the measured liquid $t=\hat{t} / L_{f}$.

Thickness calibration was performed using a custom aluminium liquid bath machined with a flat, constant gradient slope. A transparent optical flat was mounted to the bath, enabling illumination and imaging of the calibration fluid. This provided a linear variation in thickness across the range $0<{ }^{450}$ $t<5.55$. This bath was filled with a sample of the doped fuel used for atomisation and images of the liquid film acquired using the ERLIF instrument. By registering the recorded images and calculating their quotient, the linear relationship between the measured ERLIF ratio and thickness may be calculated, as described by Hidrovo \& Hart (2001).

Figure 6 shows downsampled data extracted from the centre of the calibration bath acquired at a range of laser pulse energies, as previous work has shown a saturation effect of the fluorescent dyes at high laser fluence Hidrovo et al. (2004).460 It is apparent from Figure 6 that the thickness recovered from any given ERLIF measurement is entirely independent of laser pulse energy, with effectively no variation in the measured ratio as the excitation laser energy increased. This indicates that the excitation fluence used for this study is insufficient to re-465 sult in the excitation nonlinearities described and therefore no additional correction is required.

Figure 6 does, however, suggest some non-linearity across the calibrated thickness range. With under-prediction of thick-

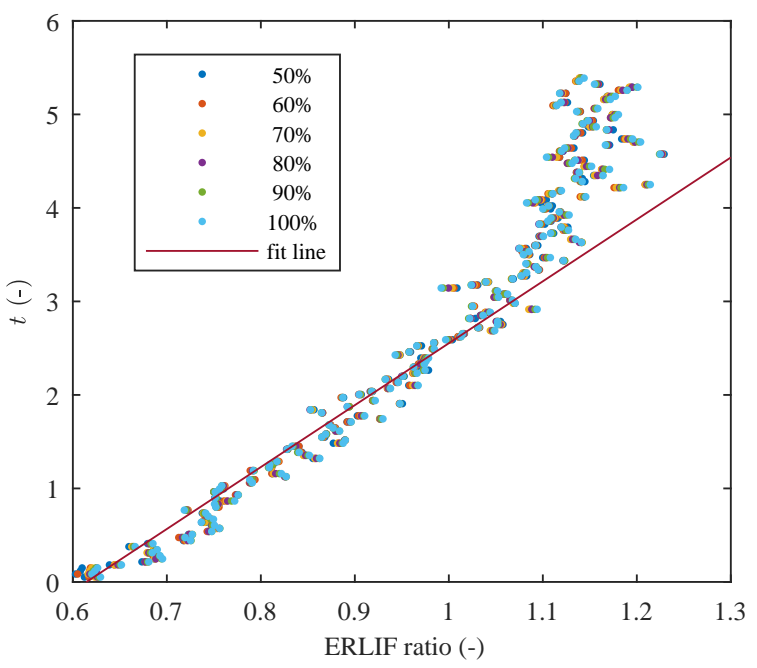

Figure 6: A typical calibration used to relate ERLIF ratio to fuel film thickness.

ness by the fitted model at higher values of ERLIF ratio being particularly pronounced. This change from the nominally linear regime $(t<3.3)$ to non-linear, is a result of the measured film becoming optically thick, as defined by Hidrovo \& Hart (2001). Under these conditions, the reabsorption tends towards invariance with additional thickness and the ERLIF technique ceases to function reliably. As previous work suggests that the flows measured on the prefilmer are expected to be thin (the work of Rizk for example found $t<2$ ), and the apparent linearity of the performed calibration across the range $0<t<3.3$, it is reasonable to perform a linear fit at low thickness and accept increased measurement uncertainties in any cases where thicker films are present. The calibration curve chosen is demonstrated by the fit line shown in Figure 6.

\subsection{Uncertainty analysis}

The uncertainty of the calibration may be estimated from the residual of the calibration bath data, in a similar manner to that performed by Les et al. (2018). The mean of these residuals provides a measure of the systematic uncertainty $\left(U_{\mu}\right)$ when the calibration shown in Figure 6 is used. Similarly, the standard deviation provides an estimate of the random uncertainty $\left(U_{\sigma}\right)$. From this the total uncertainty $U_{T}=\sqrt{U_{\mu}^{2}+U_{\sigma}^{2}}$ within the $95 \%$ confidence interval may be determined. These were found to vary with the thickness of the film and are tabulated across various ranges in table 2 . The total uncertainties calculated are higher than those claimed by Hidrovo \& Hart (2001), with total uncertainty in the region of $50 \%$ of measured value for the majority of the fuel films measured $(t<2.77)$. Because the total uncertainty at this thickness is dominated by the random uncertainty, the mean random uncertainty decreases for large numbers of samples through standard error. This is shown in Table 2 by calculating the total uncertainty for the mean of 1000 samples $\bar{U}_{T_{1000}}$. For thickness in the typical range of the measurements presented here $(t<2.77)$, the uncertainty of the average of a typical number of samples is sufficiently small for de- 
470 tailed trends within the liquid film to be measured. For thicker regions of the film, where $t>2.77$, the uncertainty increases 500 significantly, such that measurements of thickness become only qualitative.

The impact of these uncertainty restrictions on the fuel thick475 ness measurements performed on the prefilmer surface may be evaluated by consideration of probability density functions of 505 thickness from the performed experiments. These distributions have been calculated omitting zero thickness and invalid data, but including only thickness data for the prefilming flows, i.e. data measured within the primary atomisation zone has been ignored. The calculated distributions have been plotted in Fig-510 ure 7 , for each of the pressure conditions considered. To aid the reader, additional lines have been plotted highlighting ranges of varying uncertainty evaluated in Table 2 . For all operating conditions, the expected value is observed to be located within the linear region of the calibration with substantial margin. Table 3515 shows the total percentage of data lying within the uncertainty bands shown in Table 2. For all test cases, over $94 \%$ of thickness is measured within the linear regime, indicating that for the vast majority of data, a quantitative thickness is returned. For the highest levels of thickness $(3.33<t<5.55)$ where the uncertainty is sufficient that the technique may only reasonably be described as thicker/thinner, relate only to a very small ${ }^{520}$ percentage of the total number of measurements. Additionally, irrespective of the uncertainty band considered, the percentage of measurements made at elevated uncertainty levels decreases as the operating condition is increased, a result of a decrease in the fuel film thickness.

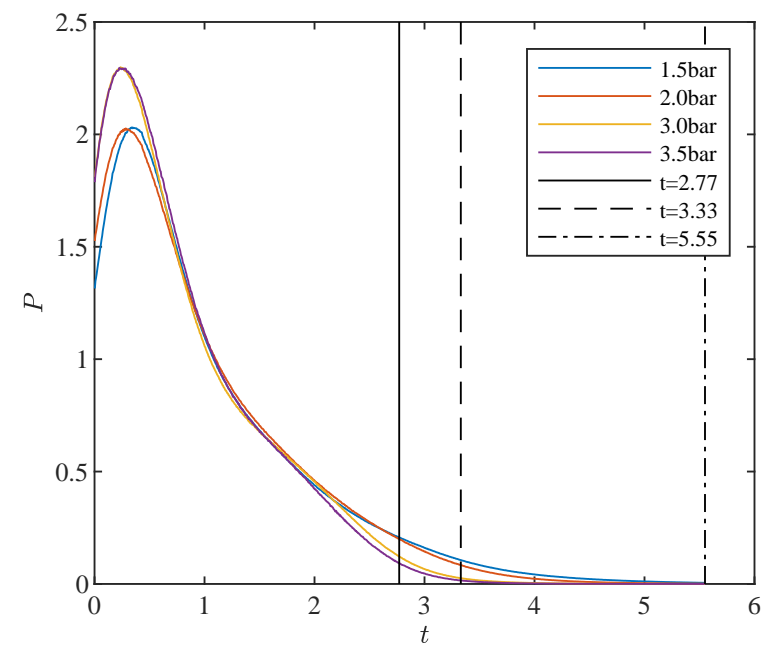

Figure 7: Probability density functions for all thickness data measured on the ${ }_{545}$ prefilmer surface.

Table 2: Uncertainty estimates

\begin{tabular}{lccccc}
\hline Thickness range & $\boldsymbol{U}_{\boldsymbol{\mu}}$ & $\boldsymbol{U}_{\boldsymbol{\sigma}}$ & $\boldsymbol{U}_{\boldsymbol{T}}$ & $\boldsymbol{U}_{\boldsymbol{T}}(\%)$ & $\overline{\boldsymbol{U}}_{\boldsymbol{T}_{\mathbf{1 0 0 0}}(\%)}$ \\
\hline $0<t<2.77$ & 0.008 & 1.145 & 1.145 & 41.3 & 1.335 \\
$2.77<t<3.33$ & 0.711 & 1.360 & 1.535 & 46.1 & 21.401 \\
$3.33<t<5.55$ & 2.907 & 2.933 & 4.131 & 74.4 & 52.446 \\
\hline
\end{tabular}

Figure 7 additionally indicates that for all pressure conditions measured, the probability density does not converge towards a probability of zero at zero thickness. This means that the measurement performed does not return thickness information for values lower than some finite measured thickness, effectively the measurement has some detection threshold. For the conditions measured here, the value of the detection threshold is $\sim 1 \mu \mathrm{m}$, with $1.5-2 \%$ of measured data of thickness smaller than $5 \mu \mathrm{m}$. It is interesting to note that for a typical good machined finish, $R a O(1 \mu m)$ may be expected. Whether this detection limit is due to the fluorescent return of the two dyes falling below the reasonable detection range for the cameras, or because the interaction of the liquid film surface tension with the prefilmer surface topology prevents the formation of films smaller than this limit is not known. In any case, the detection threshold is orders of magnitude lower than the mean values of thickness measured, hence any cumulative error from its omission in the presented statistics will be small.

\subsection{Data analysis}

To recover the thickness of the liquid film from the measured fluorescent return, some data analysis effort is required and the process followed illustrated by Figure 8 . The objective is to recover fuel thickness information on a coordinate system defined by the prefilmer geometry.

Initially, the recorded fluorescence images were registered using DaVis 8.2 by means of the geometric calibration described above. The ratio of the registered images was then taken, allowing the ERLIF calibration to be applied and outlier detection to be performed using an in-house Matlab code. Outliers were identified in two ways. For cases where no signal was returned by the fluorescence images, (defined by a threshold of 5305 counts to account for the sensor noise floor), the measured film thickness was below the detection limit of the instrument and set to zero. During the experiments described, the laser energy was attenuated such that the maximum returned fluorescent intensity measured was well below the saturation limit of the sensor. In order to prevent any unpredicted events from biasing the accumulated statistics, any occurrences of saturation were also identified (defined by a threshold of 255 counts) and marked as NaN. Despite this, unphysical values for film thickness were occasionally measured. Downstream of the prefilmer tip, some small residual registration error $(\sim 1 p x)$ was identified between the fluorescent images acquired by the two cameras. This offset results in a mismatch in the location of a given structure measured by the two cameras used to perform the thickness measurement. Because of the high spatial gradients of fluorescent return within this region, large variations in the measured thickness can therefore result from this offset.

Table 3: Percentage data within uncertainty range

\begin{tabular}{lcccc}
\hline Thickness range & 1.5 bar & 2.0 bar & 3.0 bar & 3.5 bar \\
\hline $0<t<2.77$ & 94.21 & 95.66 & 98.36 & 98.90 \\
$2.77<t<3.33$ & 3.11 & 2.77 & 1.28 & 0.90 \\
$3.33<t<5.55$ & 2.68 & 1.57 & 0.36 & 0.20 \\
\hline
\end{tabular}


Recorded images
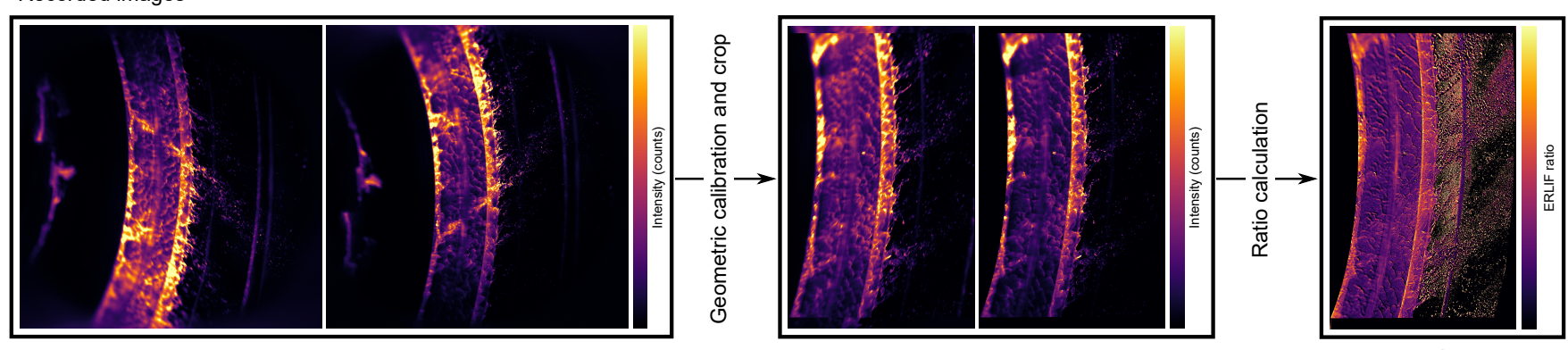

Thickness calibration and outlier de
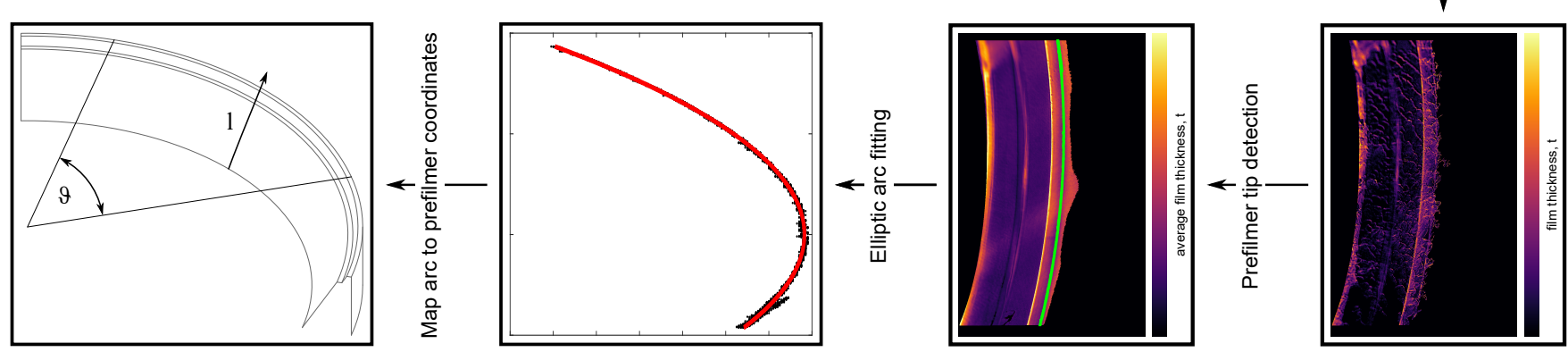

Figure 8: An illustration of the method used to process recorded fluorescence images to recover fuel thickness in prefilmer coordinates.

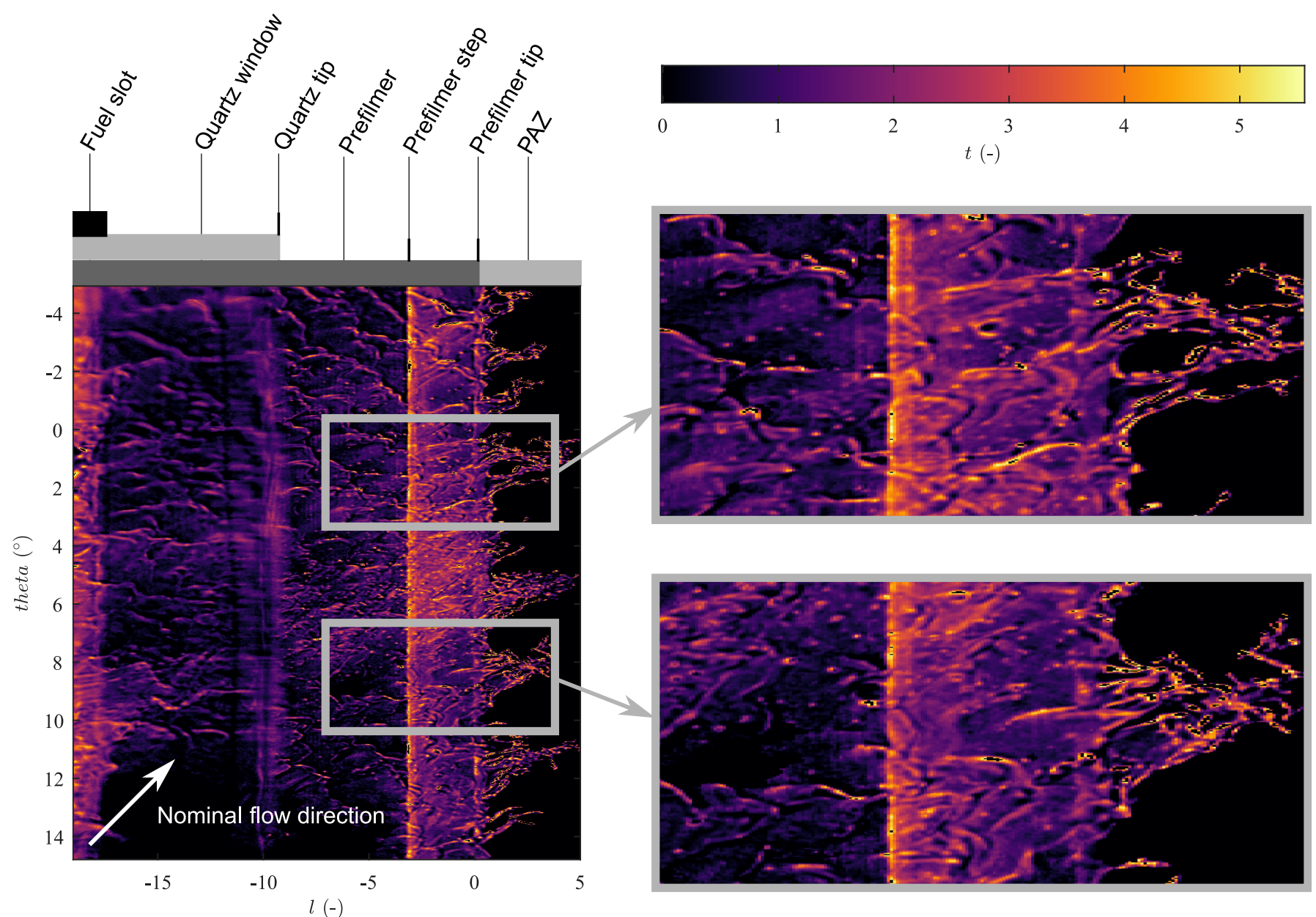

Figure 9: A sample reconstructed thickness field, highlighting the absence of thickness changes as fuel leaves the prefilmer tip. 
By filtering thicknesses that lie outside of the calibrated range $(t>5.55)$ and setting their values to $\mathrm{NaN}$, such artefacts were ${ }_{605}$ eliminated.

The time-average of all samples was then calculated and image analysis performed to identify the tip of the prefilmer. By calculating spatial gradient of thickness in the horizontal image direction, the tip could be reliably identified by manual thresh-610 olding. The prefilmer tip coordinates where then iteratively regressed to a model of an elliptic arc using non-linear least squares and a gradient descent optimiser. Because the imaging system used is of long focal length and is at large working distance from the object plane, the image projection is reasonably modelled by weak perspective. Under these conditions, the projective transform of the circular prefilmer tip becomes615 affine. This means that off-axis viewing of a the prefilmer tip will result in an ellipse being imaged. Where a segment of the prefilmer tip is imaged, as is the case here, the formed image will be that of an elliptic arc. Thus the regressed model allows definition of a transform to map the imaged data onto prefilmer ${ }_{62}$ space, with the horizontal direction corresponding to the length coordinate along the prefilmer $l$, and the vertical direction, the angular coordinate of the circular base of the conical prefilmer. The prefilmer length coordinate origin was chosen as the prefilmer tip, so that $l<0$ corresponds to regions of flow on the ${ }_{625}$ prefilmer and $l>0$ corresponds to regions downstream of the prefilmer. A sample reconstructed image is shown in Figure 9 with relevant features indicated. The formation of the prefilmer tip, prefilmer step and fuel slot as straight, vertical lines within projection across the field of view.

As outliers in the calculated thickness fields were marked with $\mathrm{NaN}$, interpolation of the thickness data onto the identified prefilmer coordinates will result in outlier propagation. To esolve this, the recorded fluorescent images were instead in-635 terpolated using the affine transform built from the thickness data. The ratio calculation, thickness calibration and outlier detection were then once again performed on this interpolated fluorescence data. This results in thicknesses being constructed

585 in prefilmer coordinates. Mapping the data to prefilmer coordi-640 nates in this way provides a convenient means of interrogating the measured data. Spatio-temporal averages may be easily calculated by integration of the transformed thickness data in the vertical and time directions to find bulk properties.

Figure 9 shows a typical reconstructed field, with the geom-645 etry observed in the scene annotated for reference and regions between the prefilmer step and tip magnified. The flow issuing from the fuel slot is observed to propagate along the main prefilmer surface as a sequence of waves. Between these waves, 595 the fuel film is extremely thin, with the possibility that the pre-650 filmer surface actually becomes dewetted and contains no film. Some distortion of the upstream portion of the prefilmer flow occurs as a result of imaging through the quartz window. Aside from the tip of this window however, the structure of the prefilmer flow remains clearly visible. As the fuel flow reaches 655 the prefilmer step the fuel film temporarily thickens, with wave structures observed crossing the region of thickening, as indicated in the high magnification sub-images of Figures 9. The flow between this step and the prefilmer tip retains an apparent wave structure, with the waves seemingly propagating directly off the prefilmer into the primary atomisation zone. As the fuel crosses the prefilmer tip, no sudden change in the thickness measured is apparent. This suggests that the ERLIF permits a first-order measurement of the thickness of the ligaments formed within the primary atomisation region.

\section{Results}

\subsection{The structure of prefilming flows}

Representative instantaneous thickness fields and ensemble averages are shown in Figures 10 and 11 respectively. As fuel flow rates have been momentum scaled from typical engine operating conditions, fuel flow rates increase at higher pressure conditions. Several bulk features are common to all of the conditions measured and may be seen in both the instantaneous thickness data and time average. In the circumferential direction, a thicker flow is observed propagating from the fuel slot at $\theta=12^{\circ}$ to the prefilmer tip at $\theta=6^{\circ}$, this is particularly pronounced in Figures 10a and 11a. The location of this structure is consistent with the location of one of the eight fuel ports located in the upstream fuel galleries and described in Section 2.1. The discrete internal fuel feed forms a jet of fuel that propagates across the prefilmer. The angle of this jet on the prefilmer is consistent with both the fuel and air nominal bulk swirl directions, which are similar for the design of the atomiser considered here. The occurrence of circumferential variation in both the instantaneous and time averaged measurements indicates that this occurs on a statistically representative basis.

In addition to the distortions on the prefilmer surface, the fuel jet also results in an increase in the delivered fuel flow into the primary zone and an associated increase in the average thickness immediately downstream of the prefilmer tip, which occurs around $\theta=5^{\circ}$ (Figure 11). Barker \& Carrotte (2014) visualised this jet with fluorescence imaging and correlated its location with localised increases in downstream fuel drop size and mass flux distribution using PDA measurements, which are shown in Figure 1. Associating the prefilmer fuel jet with a change in fuel film thickness which propagates into the primary atomisation zone is significant. It indicates that the thickness of the prefilmer fuel flow can influence the downstream droplet size distribution.

In the ensemble average fields shown in Figure 11, thicker regions are also observed to extend into the primary atomisation zone, at circumferential locations outside of the prefilmer jet. These are located at $\theta=12^{\circ}$ and $\theta=-4^{\circ}$ for all conditions measured (Figure 11). The angular spacing of these features approximately matches the angular spacing of the swirl vanes within the outer passage, which contains 22 turning vanes $\left(16.36^{\circ}\right.$ spacing). This contrasts with the inner passage which air blasts the prefilmer surface and contains just 18 turning vanes $\left(20^{\circ}\right.$ spacing). Ligaments are still shed into the primary atomisation zone along the entire circumference of the prefilmer tip (Figure 10), suggesting the atomisation associated with the outer passage vane flow is more frequent or extends further into the outer passage. 
Barker \& Carrotte (2014) measured variations in drop size fuel flux distribution at the spacing of the inner and outer passage vanes (Figure 1). The interaction with the inner passage vane wakes is unsurprising. The link between the KelvinHelmholtz instability forming prefilmer waves and the gas 720 phase vorticity thickness is well established Fuster et al. (2013).

665 The presence of highly turbulent vane wake structures will doubtless influence the vorticity thickness and so, therefore, the wave structure. This does not account for the correlation of primary atomisation zone structures with the periodicity of outer passage vane numbers however, the cause of which will be re-725 visited in Section 3.4.

The instantaneous fields in Figure 10 show small scale chaotic wave peaks on the prefilmer surface that are not present in the mean fields. Wave peaks are observed crossing the prefilmer, appearing as lines in the field which are oriented nom-730 675 inally orthogonal to the fuel jet direction. Outside of the fuel jet, upstream of the prefilmer tip, the waves are typically isolated with regions of zero measured thickness separating the wave structures. This means that outside of the prefilmer fuel jet, the prefilmer fuel flow is repeatedly below the ERLIF detec-735 680 tion threshold, with the prefilmer surface potentially becoming dewetted. This means that the majority of fuel flux across the prefilmer occurs within the fuel jet.

Whilst the wave structure is nominally orthogonal to the fuel jet, the waves in fact propagate closer to the $\theta$ direction and the 740

685 fuel jet closer to the $l$ direction. Given the lower swirl of the fuel stream than the air flow through the prefilmer passage, it is reasonable to assume that the circumferential velocity of the wave flow is higher than that of the fuel jet because it is predominantly blown by the air stream. This is indicated by the ${ }_{745}$ trajectory of the wave fronts and confirmed by the thin regions between these. Likewise the closer alignment of the fuel jet trajectory to the $l$ direction is explained by a greater proportion of the fuel jet momentum originating from the internal fuel feed.

In the $l$-direction, some features are also observed for all operating conditions (Figures 10 and 11). Fuel flows across the prefilmer surface are initially thin, but thicken substantially as the fuel crosses the prefilmer step. The level of thickening measured is particularly high for the 1.5 and 2.0 bar conditions, with $_{755}$ thicknesses outside of the quantitative range for the ERLIF setup (see section 2.5). Flows between the step and tip remain thicker than on the upstream main prefilmer surface. After the fuel flow has crossed the prefilmer step, the prominence of the fuel jet is noticeably reduced. The jet remains apparent, par- -760 ticularly in the average fields shown in Figure 11, however it is much less pronounced than on the main surface of the prefilmer. The increase in circumferential uniformity as fuel flows across the prefilmer step indicates that there is some circumferential redistribution of fuel.

As the pressure set-point of the experiment (and hence the fuel mass flow rate) is increased, several features are observed to change. Most noticeable is the decrease in thickness of fuel flow as the operating pressure increases. This is seen in the instantaneous fields and average fields, (Figures 10 and 11 re-770 the operating conditions are momentum flux scaled, increasing operating pressure, results in increased fuel mass flow rate. This increase in fuel mass flow rate, combined with the decrease measured in thickness, means the velocity of the fuel film must have increased with pressure condition.

\subsection{Bulk evolution of the prefilmer flow}

In order to quantify the differences associated with changing operating condition, bulk properties of the film have been considered as a function of $l$. Because of the mapping to prefilmer coordinates, circumferential averaging is achieved by calculating the vertical average of the data presented in Figure 11 , termed $\bar{t}$. This acts to consolidate any circumferential variation arising because of aerodynamic affects resulting from the turning vanes or the fuel jet, enabling direct comparison of bulk behaviour between test conditions. Because of the very large number of samples in the vertical direction (in excess of 1400), the random uncertainty for each of the measured conditions is negligible and the total uncertainty dominated by the systematic uncertainty. Where the film height is thin, the total uncertainty is therefore small, and where the measured film is thick $(t>3)$, the uncertainty is large. Under these conditions, ERLIF makes only a qualitative measure of thickness, i.e. thicker, thinner may reasonably be inferred. For the measurements presented here, this is confined to the prefilmer step.

As the prefilmer surface is stepped, it is useful to consider the height of the film relative to the surface of the prefilmer $h$. This has been plotted in Figure 12 along with the prefilmer geometry, shown in grey. Downstream of the prefilmer tip, where the fuel extends beyond the atomiser into the primary atomisation zone, the thickness is plotted relative to a theoretical reference height $(h=0)$, indicated by the dashed line in Figure 12. The height of the film is distinct from the film thickness, showing instead the surface of the film relative to the prefilmer surface. The thickness is therefore the distance between the plotted data and prefilmer surface in Figure 12. Should the film height follow changes in prefilmer geometry, the film is of constant thickness. Where the prefilmer geometry changes but the film height remains constant, the surface of the film is unaffected by changes in the prefilmer surface.

Several features identified from the mean thickness fields discussed above are also observed in the circumferentially averaged film thickness curves and so represent the bulk behaviour on the prefilmer surface. As the pressure set point is increased, the bulk thickness decreases across the entire length of the prefilmer surface. Likewise as the fuel passes the prefilmer step, significant thickening is measured. For the lower pressure conditions $(P<2.5$ bar $)$, an average thickness of $t>3$ is measured and therefore the thickening immediately across the step is likely overestimated by the ERLIF measurement. As the pressure set point is increased, the measured thickness decreases, though the surface height profile does not change. This suggests that the prefilmer step likely retards the flow of fuel, resulting in some significant local thickening.

After the fuel has undergone this transient thickening associated with the step, on average, the film height returns to that measured upstream of the step. This suggests that after the 

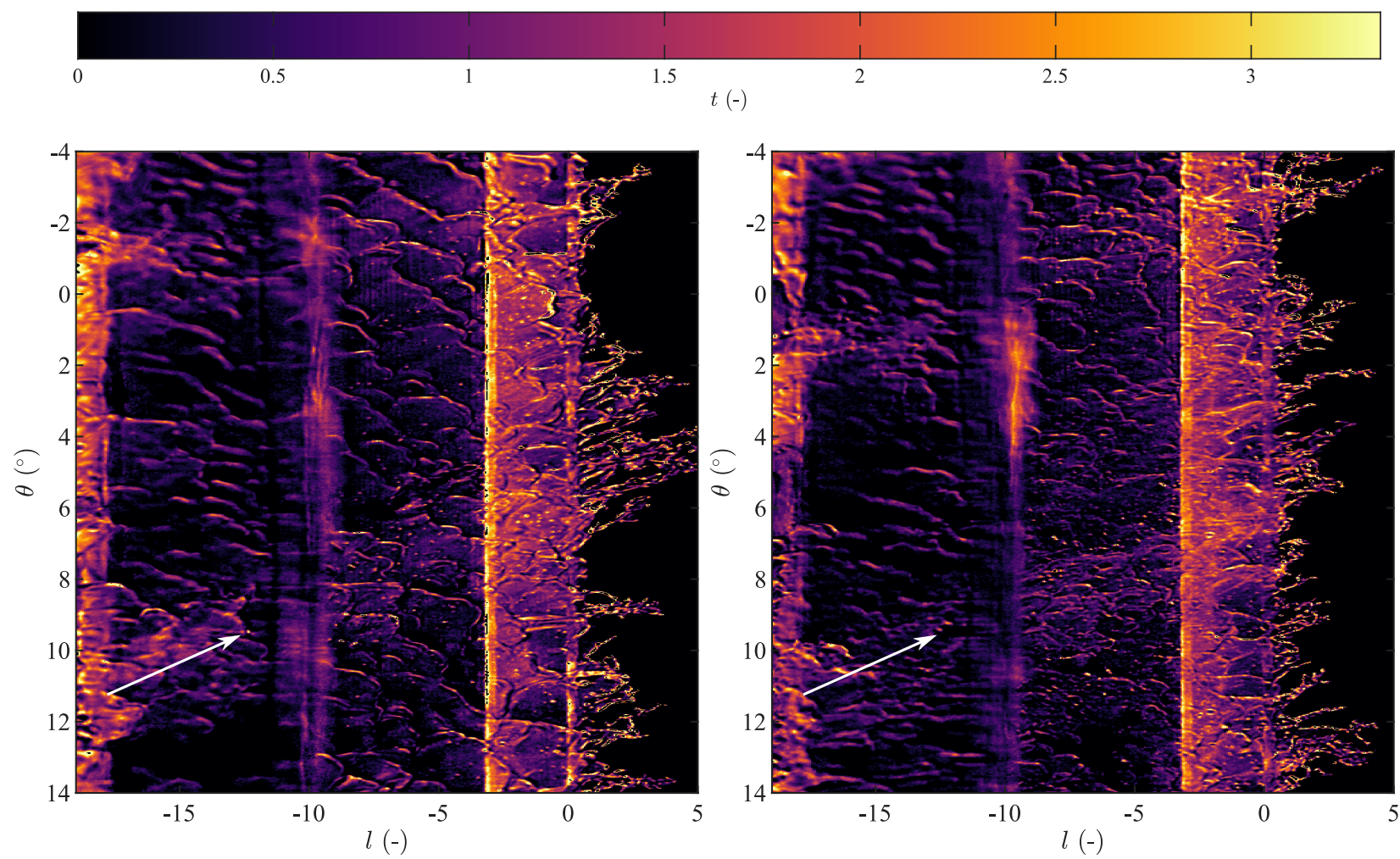

(a) $1.5 \mathrm{bar}$

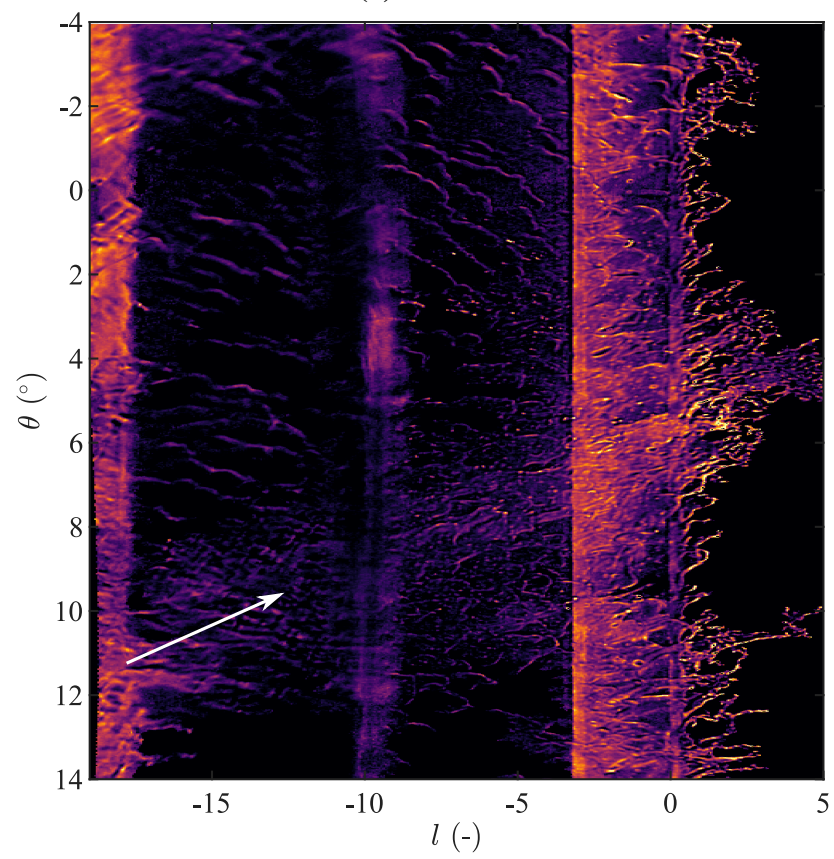

(b) $2.0 \mathrm{bar}$

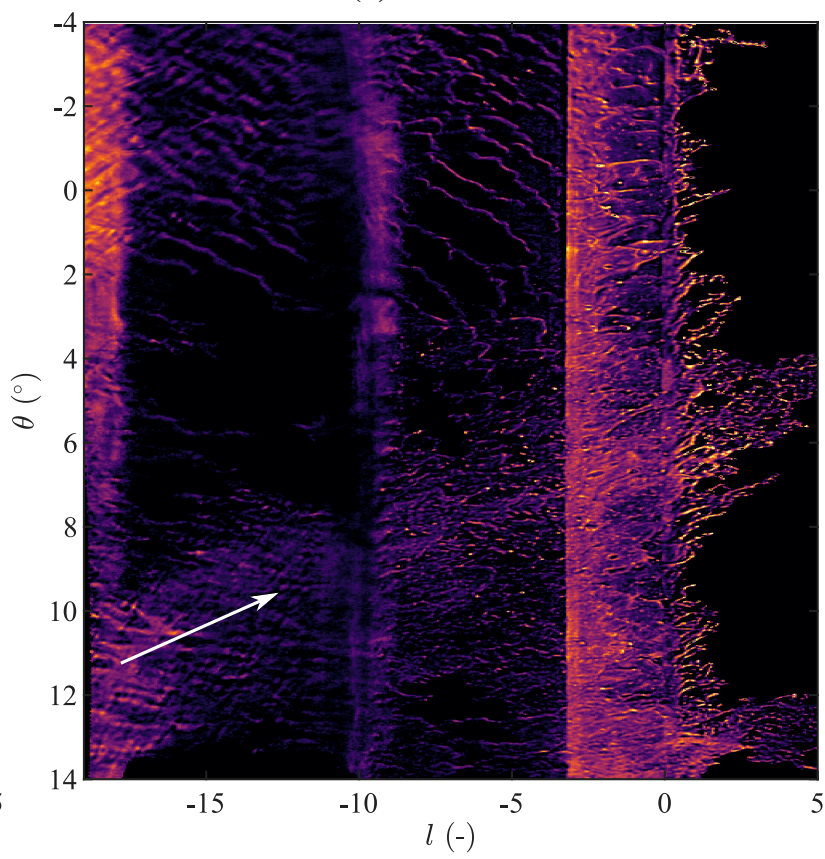

(c) $3.0 \mathrm{bar}$

(d) $3.5 \mathrm{bar}$

Figure 10: The variation of instantaneous film thickness with operating pressure. White arrows mark the location of the prefilmer jet. COLOR TO BE USED IN PRINT 

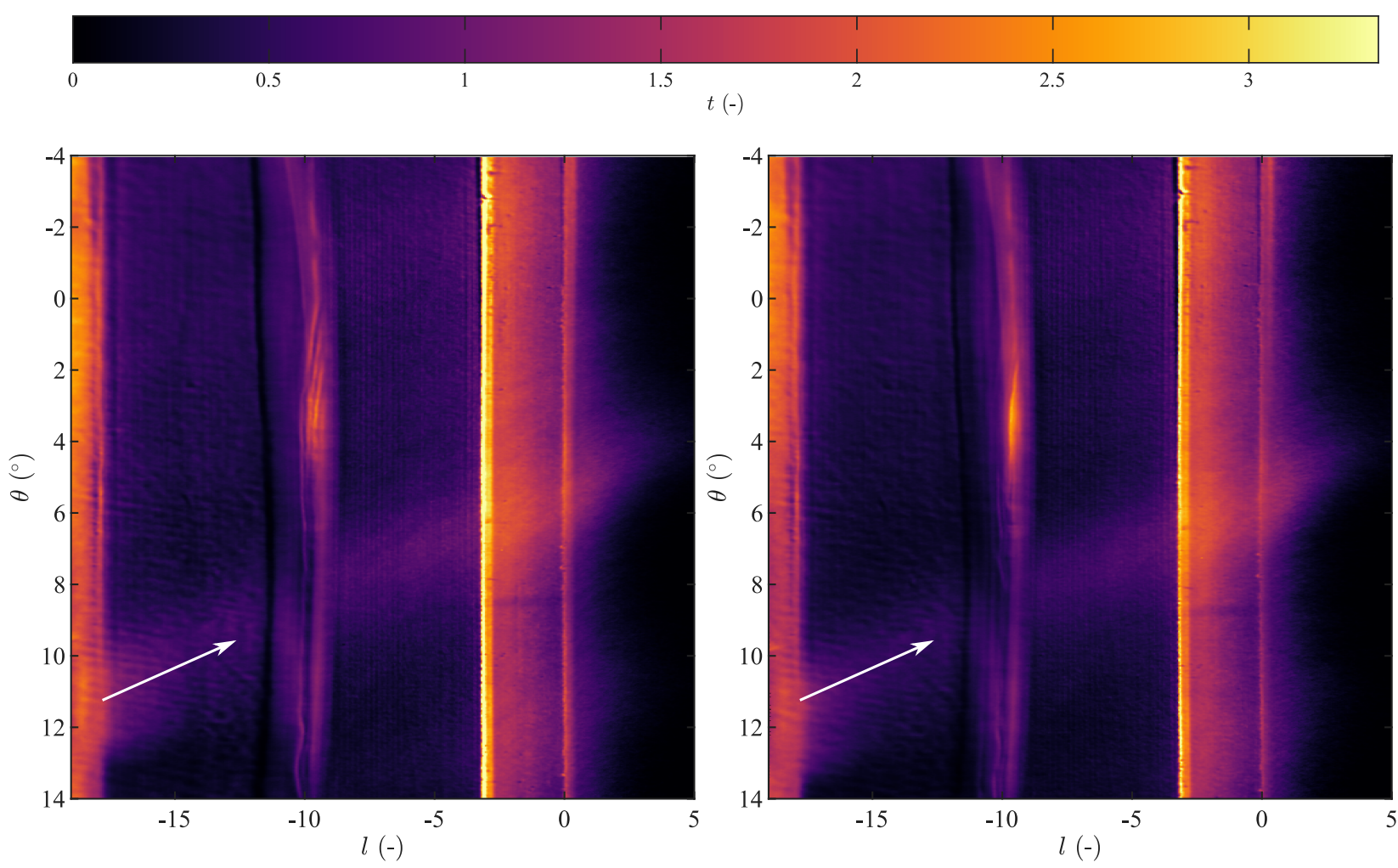

(a) 1.5 bar

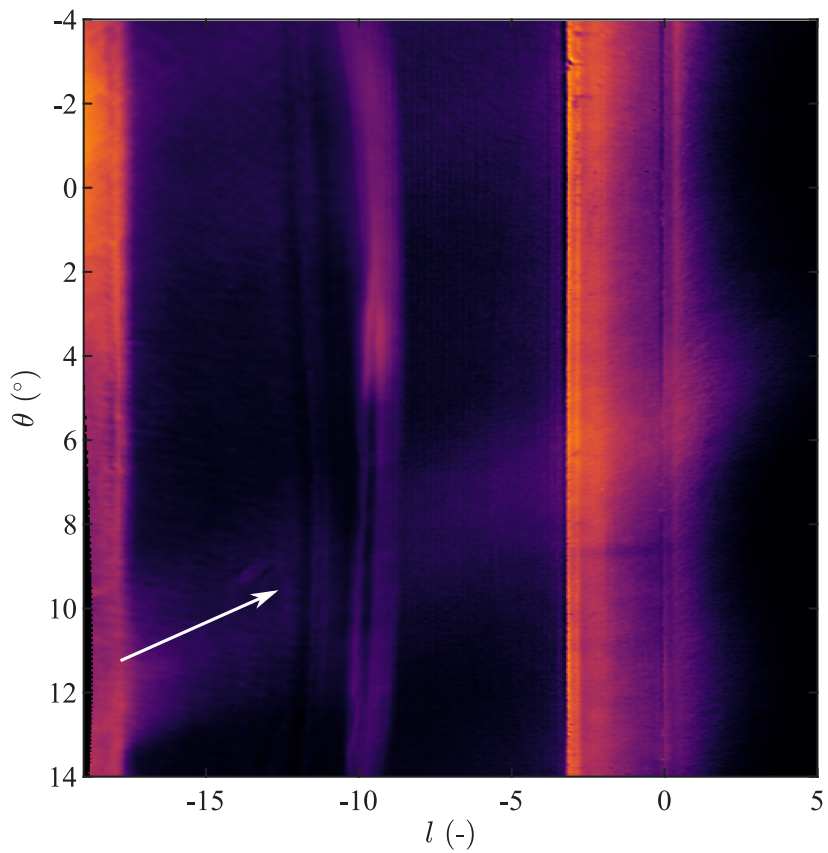

(b) 2.0 bar

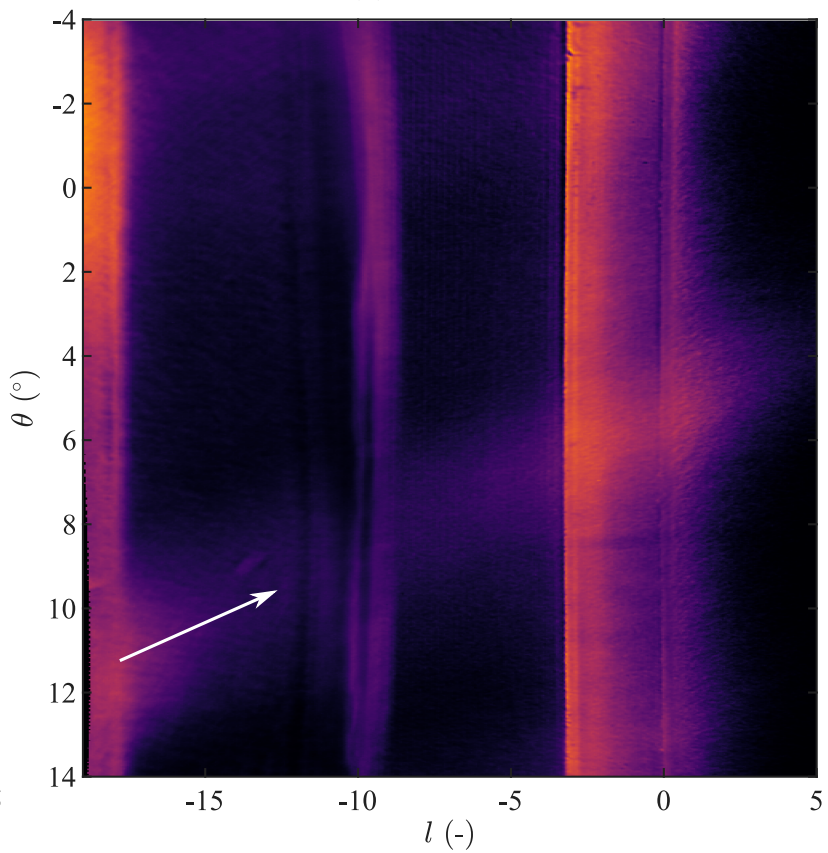

(c) $3.0 \mathrm{bar}$

(d) 3.5 bar

Figure 11: The variation of average film thickness with operating pressure. White arrows mark the location of the prefilmer jet. COLOR TO BE USED IN PRINT 


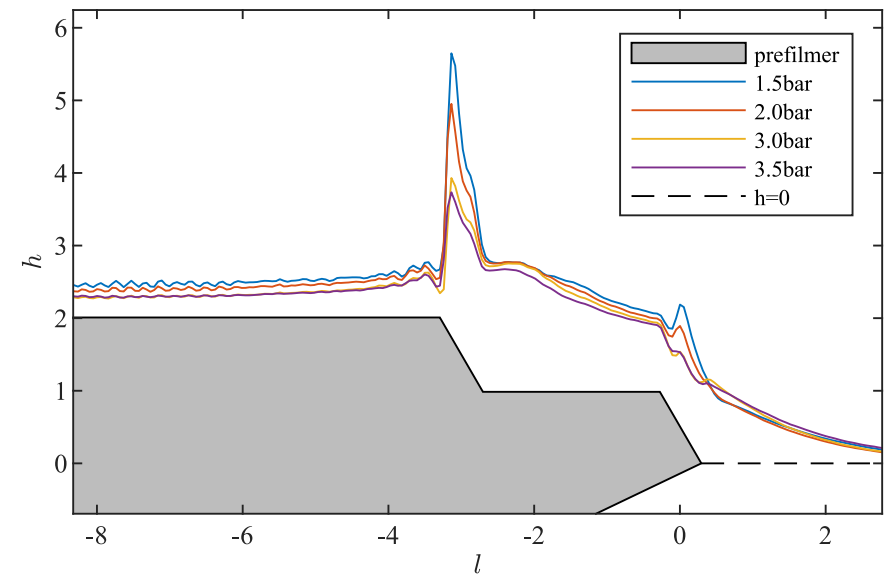

Figure 12: Circumferentially averaged film thickness, shown as film height above the prefilmer surface.

transient due to the step, the fuel returns to its original level. It forms a flow structure that retains a volume of fluid with long residence time. Instantaneous measurements across the prefilmer step support this, showing that as the wave structures propagating across the step boundary, two occurrences are observed: either the waves of fuel flow into the step region, supplying fuel to the volume retained; alternatively the waves flow across the step, in doing so extracting fuel from the volume retained. Examples of this are shown in Figure 13, which shows high resolution instantaneous measurements of film thickness across the step boundary at various $\theta$ locations. In these images, continuous lines associated with the prefilmer waves are observed crossing the prefilmer step. Where this occurs, additional fuel is collected and transported behind the wave front.

Thickness data may also be used to calculate the bulk veloc- ${ }^{810}$ ity of the fuel flow as a function of $l$. An estimate of the bulk velocity $U_{f}$ may be made from the fuel mass flow rate $\dot{m}_{f}$ and the sectional area of the fuel film by continuity. The sectional area of the film was calculated from the height of the prefilmer from the atomiser axis $h_{p}$ and the circumferentially averaged ${ }^{815}$ film thickness. Rearranging gives (3). Formulation of a bulk velocity in this way assumes that all mass flux is transported through the sectional area defined by the film thickness.

$$
U_{f}=\frac{\dot{m}_{f}}{\pi \rho_{f}\left(2 h_{p} \bar{t}-\bar{t}^{2}\right)}
$$

Bulk velocity data is shown in Figure 14 with varying pressure set point. A consistent trend is observed across all test data with fuel bulk velocity increasing as the pressure set point is increased. This occurs because the test conditions are momentum flux scaled, hence increased pressure results in increased fuel mass flow rates. It may be seen from Figure 12 that increasing the pressure set point additionally results in a decrease in fuel thickness. As the fuel film becomes thinner, it follows that the sectional area of the film must also reduce and so for constant fuel mass flow rate, the fuel bulk velocity should increase. As the fuel mass flow rate is actually momentum flux scaled, this affect is compounded, resulting in greatly increased bulk velocities with pressure set point. The affect of momentum flux scal-

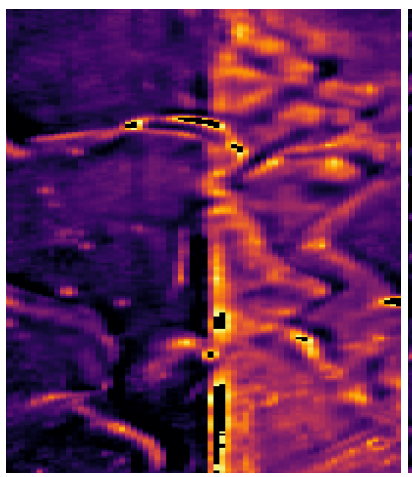

(a) $\theta=-1.5^{\circ}$

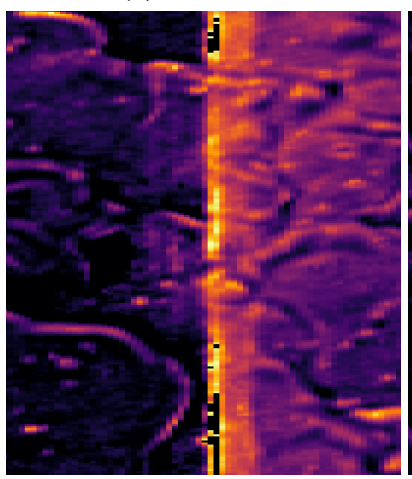

(c) $\theta=8^{\circ}$

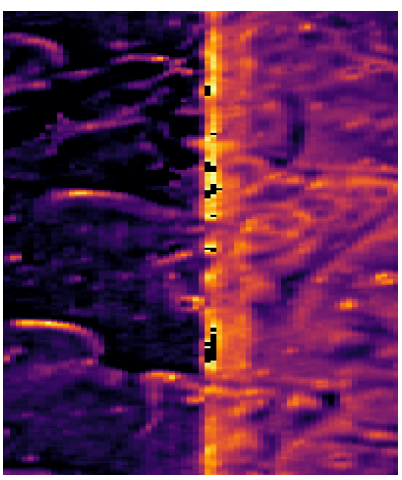

(b) $\theta=6^{\circ}$

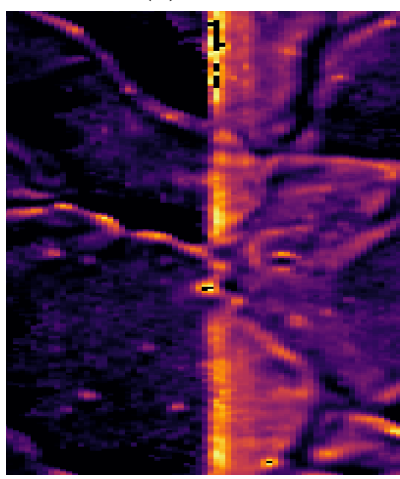

(d) $\theta=12.5^{\circ}$
Figure 13: Zoomed views of fuel thickness across the prefilmer step, highlighting the propagation of waves of fuel across the step, 1.5bar test condition.

ing engine conditions, in particular to ambient conditions, has potential applications for experimental design of representative experiments. Assuming no change in sectional area, the bulk velocity of a modest power 10bar operating condition should be 3.16 times higher than an equivalent momentum flux scaled test at atmospheric pressure. Given the consistent affect of decreasing thickness with increasing pressure, it is reasonable to expect that the real bulk velocities are greater than 3.16 times the equivalent atmospheric test.

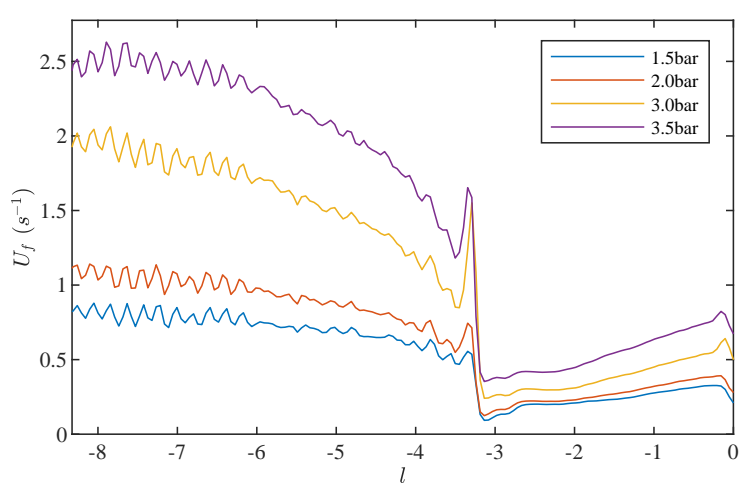

Figure 14: The bulk velocity of the fuel film, shown as a function of prefilmer length.

The bulk velocities also show consistent behaviour in the $l$ direction with pressure. As the fuel approaches the prefilmer step, 
the fuel decelerates, consistent with the thickening observed in some fuel is retained by the prefilmer step, the assumption that 870 all mass flux is transported through the sectional area defined by the film thickness is likely invalid. It has already been demonstrated that waves crossing the prefilmer step entrain retained

825 fuel, transport this across the step to tip region, increasing circumferential uniformity. Hence, close to the step, it is reason-875 able to expect that the thickness measured is a combination of both retained (possibly recirculated) fuel and fuel that flows downstream towards the tip. This implies an overestimate of

${ }_{830}$ the sectional thickness and so an underestimate of the bulk velocity.

\subsection{Prefilmer tip flows}

This hypothesis is considered theoretically, modelling the prefilmer as a simple backwards facing step or corner. The basis of the model is simple; for a liquid film to flow around a corner, the film must change direction. This acceleration must be driven by a force, surface tension.

Several authors have considered the conditions required for ${ }^{885}$ a thin liquid film to accelerate around a corner, developing and validating 1D models Owen \& Ryley (1985); Friedrich et al. (2008); Bacharoudis et al. (2014). The modelling approach used constructs a force/momentum balance across the presented corner: where the forces imposed by surface tension and gravity are sufficient to entirely overcome the liquid film momentum, the flow is accelerated around the corner; where momentum dominates these forces, the film does not flow around the corner, but instead will detach from the solid surface. In order to define this force ratio, the balance is defined in the direction of flow before reaching the corner, the $l$ direction for the atomiser considered here.

For the prefilmer flow, instantaneous images suggest that it is the occurrence of wave peaks at the prefilmer tip that motivate the formation of ligaments and so initiate the atomisation process. The model presented by Bacharoudis et al. (2014) was therefore evaluated, as the inertial affects of both the bulk film and the wave flow are considered. For application to prefilmer flows, the force ratio resulting in fuel detaching, or accumulating at the step, is simplified by the orientation of the region of the prefilmer measured. In the plots shown in Figures 9-

${ }_{860} 11$, gravity is directed downwards, orthogonal to the direction of the net surface tension force and the $l$ direction, so may be eliminated from the force balance. The force ratio, with the gravitational terms omitted is given by (4) and is proportional to the prefilmer Weber number $W e_{p}(5)$.

$$
\begin{gathered}
F R=\frac{\rho_{f} h_{w} c_{w}^{2}}{\sigma_{f} \sin (\beta)} \frac{L_{w}}{4\left(R_{c}+h_{f}\right)} \\
W e_{p}=\frac{\rho_{f} h_{w} c_{w}^{2}}{\sigma_{f} \sin (\beta)}
\end{gathered}
$$

The measurements performed may be used to bound the values of force ratio expected for the prefilmer tip, determining ${ }_{900}$ whether the liquid film is in a momentum or surface tension dominated regime. The corner radius $R_{c}$ of the atomiser is sharp $\left(R_{c}=0\right)$ and the angle between the prefilmer surface and the face of the prefilmer tip $\beta$. The thickness of the unperturbed film is measured from circumferentially averaged thickness data, $h_{f}=\bar{t}(l=0)$. The wave motion is modelled by the wave prominence $h_{w}$, the wavelength $L_{w}$ and the wave speed $c_{w}$. Following Bacharoudis et al. (2014), the height of the wave may be estimated from the standard deviation of the measured thickness data (6).

$$
h_{w}=2 \sqrt{2\left\langle\hat{t}^{\prime 2}\right\rangle}
$$

Because the instantaneous measurements show waves extending from the prefilmer step to tip, this length is used to define $L_{w}$. The wave speed is less straightforward to determine as this is not directly measured. A conservative estimate is desirable, as $W e_{p}$ and so $F R$ scales on the square of wave speed. As the liquid film is thin and shear driven, Couette flow provides an approximation of the velocity distribution in a uniform film. The velocity of the surface of such a flow being twice the bulk velocity, which may be determined from Figure $14, c_{w}=2 U_{f}(l=0)$. The wave dominated flows on the prefilmer are clearly not spatially or temporally uniform and therefore defining $c_{w}$ in this way will underestimate the true wave speed on the film. Despite this underestimation of the film momentum, the calculated force ratio should remain appropriate to an order of magnitude and so determine whether the prefilmer flows are expected to be inertia dominated, surface tension dominated or balanced.

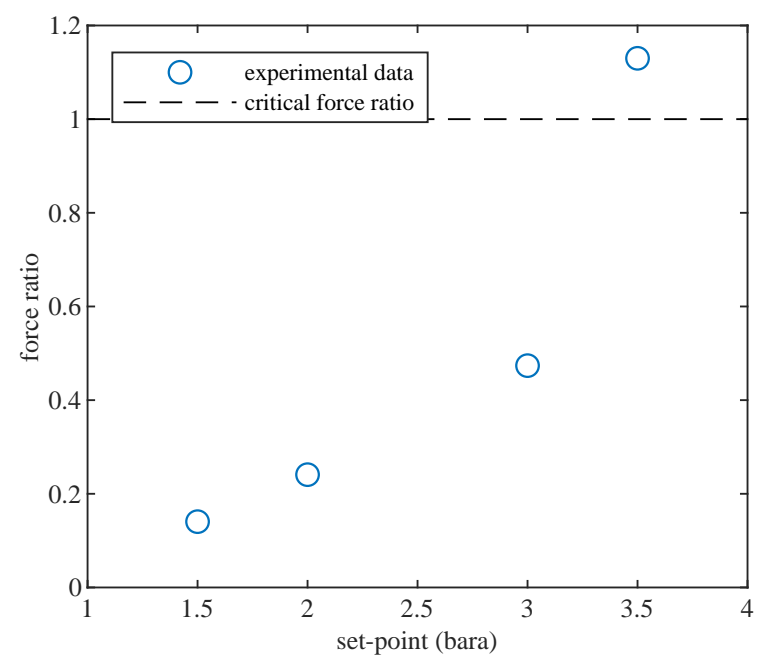

Figure 15: Force ratio by operating condition

Estimates of the force ratio calculated from (4) are shown by operating condition in Figure 15, with separation of the film predicted for force ratios exceeding unity. The comparison with observations from the bulk measurement of the experiments performed here is reasonable. Figure 15 shows increased momentum effects at higher pressure conditions and increased surface effects tension at lower pressure conditions. At all conditions, however, the force ratio is on the order of unity, 
indicating that neither momentum nor surface tension could be expected to dominate the flows at the prefilmer tip. The 960 flows could therefore be considered to be in transition between surface tension and momentum dominated regimes, with both atomisation events taking place.

The identification of the importance of the momentum, surface tension balance at the prefilmer tip and the ${ }_{965}$ parametrisation by $W e_{p}$ is significant, explaining the physics which determine whether downstream drop size is correlated with prefilmer film thickness. For a given prefilmer geometry, this is defined by the relative magnitude fuel momentum and surface tension. Where film momentum is low, surface tension may cause all of the fuel to accelerate onto the prefilmer tip prior to atomisation. Under these conditions, the outer length scale of the ligaments and so the downstream drop size distribution will be defined by the thickness of the prefilmer tip, independent of the thickness of the liquid film. If the same geometry were to be operated at increased fuel momentum, surface tension will be insufficient to turn the fuel onto the tip and it will, instead, flow directly into the primary atomisation zone. In this case, the outer length scale for the downstream drop size distribution will be defined by the thickness of the fuel film, and so drop sizes will be correlated with fuel film thickness. It is intuitive to think of the behaviour at the prefilmer tip as setting the boundary conditions for the subsequent primary atomisation process. Primary atomisation is well known to be parametrised at leading order by the Weber number (1). Changes to $W e_{p}$ (5), however, influence the relevant length scale of the ligaments presented to the primary atomisation zone, defining $L_{f}$ in the Weber number (1).

Because the wave speed influences $W e_{p}$ and wave transport is by definition an intermittent process, it is a local, instantaneous force balance that will determine the behaviour of the liquid film at the tip. Given the interaction between the film and the turbulent air stream, it is reasonable to assume that the wavelength and wave velocity vary stochastically in both time and space. Inspection of the instantaneous measurements reveal significant variations in the thickness of the fuel to the 40 imaged region of the prefilmer, suggesting either large variations in the local velocity of the film. This means that primary atomisation events may occur at both high and low $W e_{p}$ on the same prefilmer at the same point in time. As there are high $W e_{p}$ atomisation events occurring, the downstream drop size distribution will therefore be correlated with the film thickness.

Figure 16 shows an instantaneous measurement of thickness across a section of the prefilmer, extending from the step into the primary atomisation zone. The fuel flows at the tip, clearly demonstrate primary atomisation occurring at high and low ${ }_{950} W e_{p}$ at the same point in time. Towards the top of the image $\left(-2^{\circ}<\theta<4^{\circ}\right)$ fuel has accumulated, being held by the prefilmer step, with localised dewetting upstream of the tip. Fuel residue is observed being retained on the tip by surface ten-970 sion forces and ligaments are seen to form. This occurs as a result of fuel accumulation on the tip. Individual waves are are also shed from the fuel retained at the prefilmer step and subsequently travel directly across the prefilmer tip. This is evidenced by Figure 16, where an isolated wave can be observed 975 directly crossing the prefilmer tip at $\theta \sim 1.5^{\circ}$. These isolated wave shedding events, may well trigger the atomisation events observed elsewhere in the low momentum zone. Additionally, large wave shedding processes are observed. Large volumes of fluid flow from the fuel retained at the prefilmer step, across the tip and are directly atomised. This is shown in the region of $4^{\circ}<\theta<6.5^{\circ}$ in Figure 16, but is observed to occur intermittently across the entire field of view.

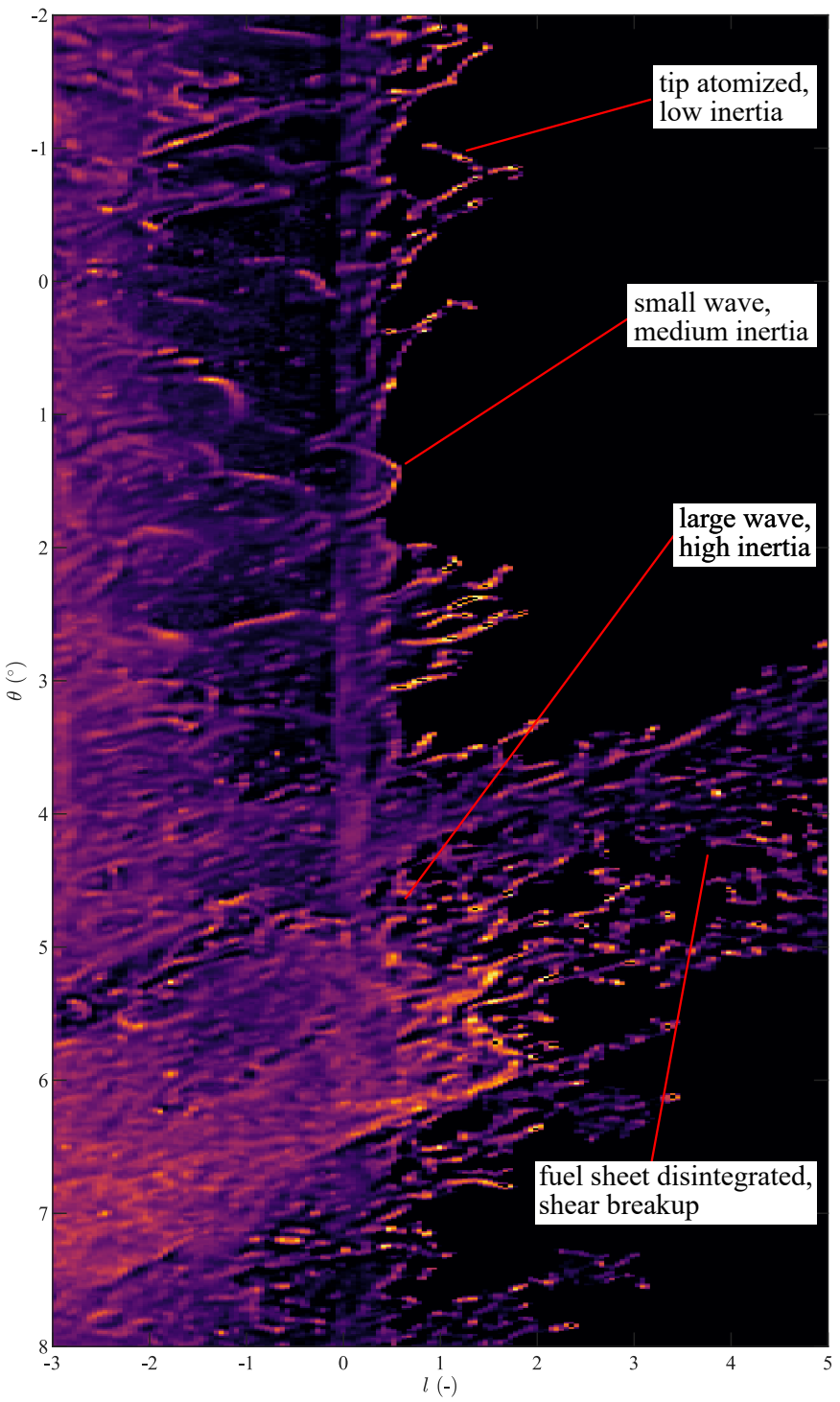

Figure 16: An instantaneous measurement illustrating the various primary atomisation mechanisms for a representative LDI atomiser, 3.5bar set-point. COLOR TO BE USED IN PRINT

Despite the circumferential redistribution of fuel caused by the prefilmer step, some circumferential non-uniformity in fuel thickness remains in the step to tip region. This is most clearly shown by the thickening associated with the fuel jet in the average fields (e.g. Figure 11d). For the lower pressure conditions tested, some bulk accumulation of fuel on the tip is suggested by the circumferentially averaged data in Figure 12, although the measured thickness increase is small compared to the size of the tip. Were there to be a constant accumulation on the tip 
across its entire breadth, a bulk increase in thickness at the tip of the order of the size of the tip would instead be seen. A relatively small increase in surface height (compared to the tip size) instead indicates that atomisation occurs through direct atomi ${ }_{+035}$ mary atomisation zone and also from fuel accumulation at the tip. For the elevated pressure conditions, only minimal increase in thickness at the tip is measured, suggesting predominantly momentum dominated flow, leading to direct atomisation dis to40 tributed around the atomiser and correlation between drop size distribution and film thickness.

In order to identify the atomisation physics associated with aerospace engine operation, the trends identified in Figure 15 are important. Considering the case of the cruise condi +045 tion, from which the measurements performed here have been scaled, the air pressures and densities are significantly higher than the test conditions presented. For a 10bar condition, the mass flow rate would be 1.69 times greater than the 3.5 bar condition considered here. Assuming constant film thickness 850 at increased pressures, the fuel momentum would increase by a factor of 2.9. In reality the fuel film thickness would be expected to decrease with operating pressure (as shown in Figure 12), resulting in further increases to the fuel momentum and so $W e_{p}$. Furthermore, gas turbine fuel is used as a coolant 055 prior to its atomisation. As a result, under engine operating conditions the fuel will have lower surface tension than in the laboratory tests performed here. The reduction in surface tension will act to further increase $W e_{p}$. This combined increase in momentum and decrease in surface tension results 060 in significantly higher values for $W e_{p}$ in the engine environment than are typically studied today. Momentum effects and so correlation between the downstream drop sizes and the fuel film thickness on the prefilmer should therefore be expected to dominate for atomisers used in gas turbine applications. This is 065 a significant finding; to best reflect engine operating conditions for studies of primary atomisation, studies should be configured to deliver high $W e_{p}$ so that fuel ligament formation occurs in a momentum dominated regime.

\subsection{Primary atomisation statistics}

Because the filtering processes implemented remove "noise" resulting from droplets in the primary atomisation region, images of the ligaments forming the primary zone are well resolved. This provides an opportunity for statistical assessment 075 of the length and placement of the ligaments formed within the primary atomisation zone and so the generation of data useful for the validation of CFD models. Regions downstream of the prefilmer are simply selected according to $l>0$. The outer limit of the liquid issuing from the prefilmer may then be 080 selected by finding areas with valid, non-zero thickness. The resulting field was then filled to remove holes and the perimeter found. Following Gepperth et al. (2012), peaks in the extracted perimeter detected as a measure of the tip of a ligament. Only peaks with a minimum width, minimum prominence and min +085 imum separation (values of 5, 10 and 3px respectively) were with variations in this parametrisation.
To provide an assessment of the statistical distribution of ligament generation, probability density functions have then been calculated from the ligament tip data. Figure 18 shows the circumferential distribution of ligaments formed, with the probability of a ligament being detected at any given location plotted. The curves show a prominent peak at $3<\theta<4^{\circ}$, with the location of this peak varying slightly with pressure set point; higher pressures exhibiting peak locations at smaller $\theta$. This location is consistent with the previous observations of the prefilmer jet penetrating into the primary atomisation zone, as shown in Figure 11 and indicates an increased level of fuel flux from the fuel jet into the primary atomisation zone.

Probability distributions of ligament length have also been plotted and are shown in Figure 19. These are cumulative statistics for all ligaments measured, as opposed to tracking the evolution of any single ligament (similar to the method used by Warncke et al. (2017)), the expected value is given by the minimum length of a shed fuel structure that may form a detectable peak. This corresponds to the peak in the probability distribution because all measured ligaments must at some point in their evolution have this length. For lengths shorter than this, the fuel film shed from the prefilmer surface is less likely to form a peak that is detectable for the applied validation conditions. The distributions computed indicate an invariance of ligament length distribution with operating condition, which is consistent with previous momentum flux scaled measurements with varying pressure set point (Bhayaraju \& Hassa, 2009).

For the 3D atomiser with swirling gas and fuel streams however, it is expected that the physical mechanism leading to atomisation differs from the planar 2D case. Because the fuel retains some tangential momentum at the prefilmer tip, as evidenced by the angle of the measured fuel jet and the measurements on prefilming swirl-cup atomisers (Shanmugadas et al., 2018), the fuel must retain some tangential momentum as it leaves the prefilmer tip. This will result in some outboard motion and was indicated in the qualitative measurements performed on the prefilmer of this atomiser, with fuel deposits detected on the outer casing of the outer passage (Barker \& Carrotte, 2014). In order to maximise shear at the prefilmer tip, the swirl number of the outer passage flow is increased relative to the inner passage. This will result in significant crossflow being applied to the fuel as it crosses the outer passage airflow. Taking the Weber number from the bulk fuel film thickness at the prefilmer tip (Table 1), the liquid flow is within the shear breakup regime (Sallam et al., 2004) for all conditions tested and this results in rapid disintegration of the fuel a large length from the prefilmer. This process is shown clearly in Figure 16 for the fuel jet.

The distance the ligaments may travel across the outer passage before the imposed aerodynamic forces become sufficient to rapidly atomise the fuel sheet is therefore influenced by the aerodynamic forces applied to the fuel structure. Because the gas flow within the outer passage passes through a vane cascade upstream of the prefilmer tip, the aerodynamics are highly complex with significant radial and circumferential variation. Multi-hole pneumatic measurements performed on an equivalent vane cascade at ambient pressure have indicated variations 
Figure 17: REMOVED

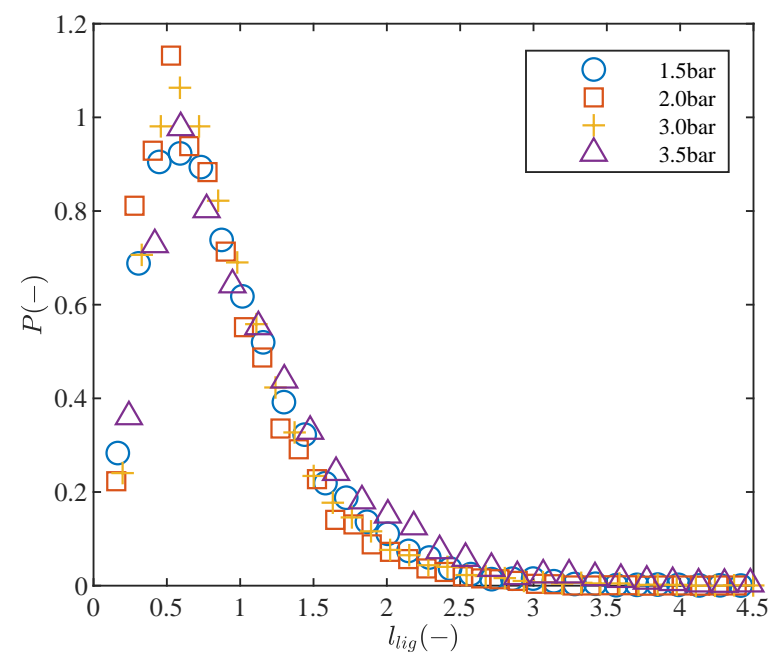

Figure 18: Probability density function, ligament circumferential position. of average total velocity of $50 \%$ at the prefilmer exit, which ing and the vane's wake (Barker \& Carrotte, 2014). Because of this spatial variation of total velocity, the location at which a fuel structure is shed from the prefilmer will influence the atomisation forces applied to it and the length it may achieve before being disintegrated. Where fuel moves into the wake of a turning vane, the aerodynamic forces applied will be lower than the case that the same fuel structure moved into the higher velocity vane core. As such, a fuel structure within the low total velocity vane wake will extend to longer lengths than an tocated in a vane core. This behaviour is also measured within the thickness fields presented here. As already noted in section 3.1, the measured average thickness is observed extending further in the $l$ direction at a spacing consistent with the separation of outer passage vanes (Figure 11) -4 and $\theta=12^{\circ}$. The circumferential distribution of measured ligaments shows a secondary peak in the region of $10<\theta<12^{\circ}$, which is consistent with the $\theta=12^{\circ}$ (Figure 18) feature in the average fields. There is evidence of a tertiary peak located at $\theta=-4^{\circ}$, however this is difficult to identify with certainty, given the level of scatter in the data.

\section{Conclusions}

This work has demonstrated the application of the ERLIF technique to the measurement of liquid films on the prefilmer of an LDI atomiser with representative aerodynamic boundary

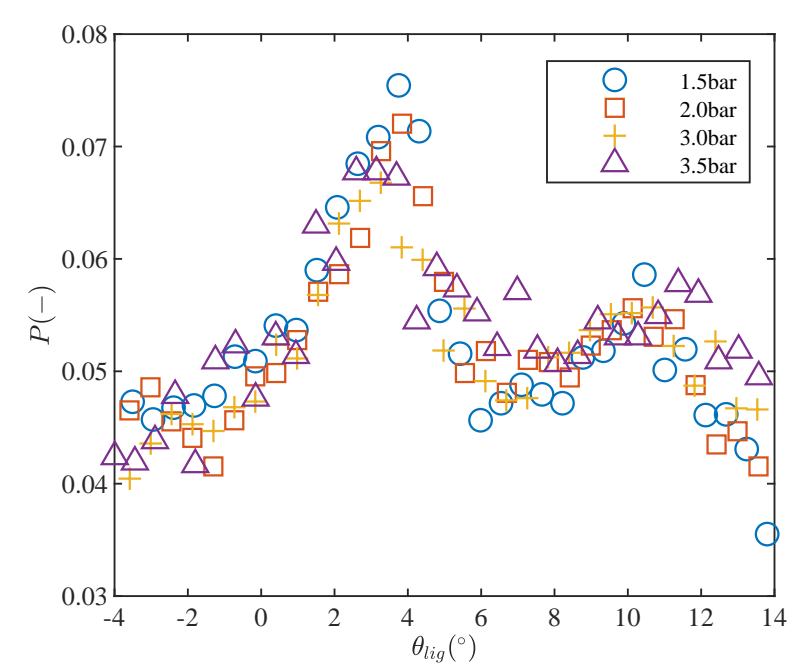

Figure 19: Probability density function, ligament length. conditions. The ERLIF instrument has been demonstrated as providing good resolution of the prefilmer flows, with the detection threshold at the limit of typical arithmetic mean roughness for good machined finish. Because the measured thickness is derived from the quotient of two fluorescence measurements, 20 any given instantaneous value has a high level of random uncertainty. For thin films, which are associated with the majority of flows on the prefilmer surface, the calibration performed shows linearity and so for large numbers of samples, the uncertainty of measured statistics diminishes due to standard error. Measurements performed on the prefilmer of the atomiser indicated no significant change in thickness as structures flowed across the prefilmer tip. This suggests that the ERLIF system provides a reasonable means of parametrising the thickness of structures within the primary atomisation region, a region currently inaccessible to point-wise measurement techniques.

The performed measurements fully describe the fuel flows for the tested atomiser. Fuel is transported across the prefilmer surface by a series of waves, with localised regions of dewetting present between wave fronts. The flow from the fuel slot is highly non-uniform in the circumferential direction, with upstream fuel passages leading to a thicker fuel jet on the prefilmer surface. Prior to reaching the tip, the tested prefilmer is stepped. This results in significant thickening, fuel retention and a redistribution of fuel in the circumferential direction. 
1140 Flows from the step to tip remain wave driven, but are significantly thicker than between the fuel slot and step. As static pressure was increased, for constant momentum flux ratio and air flow Mach number, the fuel film thickness20 decreased. Comparisons with previous measurements indicated 145 that changes to the drop size distribution were influenced by the presence of the fuel jet, suggesting correlation between fuel film thickness and drop size.

Observations of waves on the surface of the prefilmer $\operatorname{sug}_{+205}$ gested that fuel was able to flow directly across the prefilmer tip, into the primary atomisation zone. Circumferentially averaged measurements across the prefilmer showed reduced accumulation of fuel at the prefilmer tip at elevated pressure conditions, indicating a change of the bulk behaviour of the prefilmer fuel ${ }_{210}$ flow at the tip. Similar observations had been made previously and attributed to the momentum of the fuel film (Bhayaraju \& Hassa, 2009). This work therefore evaluated a theoretical model (Bacharoudis et al., 2014) to identify whether the bulk fuel film contained sufficient momentum to overcome a surface 21 tension driven acceleration at the prefilmer tip. This showed the operation of the atomiser to likely lie within a transitional region. Momentum was of the same order as surface tension forces, defined by the prefilmer Weber number, $W e_{p}$.

It is changes in this prefilmer Weber number that separate 22 examples where all fuel was able to accumulate at the tip, leading to an invariance of drop distributions with prefilmer fuel thickness (Sattelmayer \& Wittig, 1986; Aigner \& Wittig, 1988; Gepperth et al., 2013; Shanmugadas et al., 2018) from the conditions where direct atomisation occurred, leading to variations in the downstream drop size with film thickDéjean et al., 2016). This conclusion is of some significance for the atomisation community, as it resolves ongoing confusion concerning the atomisation physics appropriate to prefilming airblast atomisers. Where prefilmer Weber number is high, downstream drop size distribution should be correlated with liquid film thickness. Where prefilmer Weber number is low however, downstream drop size distribution will be independent of liquid film thickness. Therefore, an atomiser may exhibit both regimes, depending upon the conditions at which it operates. The prefilmer Weber number may therefore be considered to define the outer length scale for the subsequent primary atomisation process, also parameterised by a Weber ${ }^{23}$ number. Whether a given atomiser generates drops whose distribution is correlated with film thickness therefore depends upon the operating conditions.

At the operating conditions studied in this work, the fuel ${ }^{24}$ film had nominally balanced momentum and surface tension forces. This resulted in parts of the atomiser exhibited surface tension dominated atomisation events, whereas other regions showed momentum dominated atomisation. The result of this, ${ }^{124}$ is that the downstream drop size distributions are correlated with the prefilmer film thickness, due to the direct atomisation associated with the high momentum flows. At engine operating conditions, the fuel momentum will increase and the surface ${ }^{1250}$ tension decrease, significantly reducing the probability of surface tension dominated atomisation. Under these conditions, the correlation between film thickness and drop size distribution should improve due to a reduction in fuel accumulation at the prefilmer tip.

Finally statistics have been presented describing the ensemble behaviour of the structures within the primary atomisation region. Probability density functions of ligament length demonstrate invariance of ligament length with momentum flux scaled pressure set point. An assessment of the liquid trajectory at the prefilmer tip suggests that this occurs because the evolution of the fuel shed into the primary atomisation zone results in its transport across the outer passage gas path, which has high momentum relative to the prefilmer passage gas and fuel flows. This effectively results in the formation of jet in crossflow conditions at high Weber number, resulting in sudden atomisation of the fuel sheet. Additionally correlations were made between the circumferential distribution of ligaments within the measurements. The expected location for ligaments to form is downstream of the fuel jet, which is significant in fuel transport across the prefilmer and can be seen penetrate further into the primary atomisation zone in the average fields presented. A secondary peak was observed in the distribution, corresponding to the location of vane wakes within the outer passage. This further suggests the importance of the aerodynamic boundary conditions for the generation of sprays representative of engine conditions.

\section{Acknowledgements}

The authors wish to thank the Technology Strategy Board for funding the measurement programme under the NADIT program (project number 101368) and Rolls-Royce plc. for supporting the following data analysis. Mr Neil Thorley, Loughborough University is acknowledged for his engineering support in delivering the experimental measurements. Dr Marco Zedda and Dr Stephen Harding, Rolls-Royce plc are thanked for their ongoing advice, support and guidance.

\section{References}

Aigner, M., \& Wittig, S. (1988). Swirl and counterswirl effects in prefilming airblast atomizers. Journal of engineering for gas turbines and power, 110, 105-110. doi:https://doi .org/10.1115/1.3240072.

Alekseenko, S. V., Cherdantsev, A. V., Cherdantsev, M. V., Isaenkov, S. V., \& Markovich, D. M. (2015). Study of formation and development of disturbance waves in annular gas-liquid flow. International Journal of Multiphase Flow, 77, 65-75. doi:https ://doi.org/10.1016/j . ijmultiphaseflow. 2015.08.007.

Bacharoudis, E., Bratec, H., Keirsbulck, L., Buchlin, J.-M., \& Labraga, L. (2014). Simplified model for the prediction of the occurrence of film atomization in corner geometries. International Journal of Multiphase Flow, 58, 325-337. doi:https://doi.org/10.1016/j.ijmultiphaseflow. 2013.10 .003$.

Barker, A., \& Carrotte, J. (2014). The impact of representative aerodynamic flow fields on liquid fuel atomisation in modern gas turbine fuel injectors. In ASME Turbo Expo 2014: Turbine Technical Conference and Exposition (pp. V04BT04A047-V04BT04A047). American Society of Mechanical Engineers. doi:https://doi.org/10.1115/GT2014-26927.

Bhayaraju, U., \& Hassa, C. (2006). Surface Wave Break-up and Propagation in Planar Liquid Sheets of pre-filming Airblast Atomisers. In International Conference on Liquid Atomization and Spray Systems (ICLASS), Kyoto, Japan. 
Bhayaraju, U., \& Hassa, C. (2009). Planar liquid sheet breakup of prefilming 325 and nonprefilming atomizers at elevated pressures. Atomization and Sprays, 19. doi:https://doi.org/10.1615/AtomizSpr.v19.i12.50.

Bremond, N., Clanet, C., \& Villermaux, E. (2007). Atomization of undulating liquid sheets. Journal of Fluid Mechanics, 585, 421-456. doi:https : // doi.org/10.1017/S0022112007006775.

Carvalho, I., \& Heitor, M. (1998). Liquid film break-up in a model of a prefilming airblast nozzle. Experiments in Fluids, 24, 408-415. doi:https : //doi.org/10.1007/s003480050190.

Chaussonnet, G., Riber, E., Vermorel, O., Cuenot, B., Gepperth, S., \& Koch, R. (2013). Large eddy simulation of a prefilming airblast atomizer. In In+335 ternational Conference on Liquid Atomization and Spray Systems (ILASS), Chania, Greece, Sept (pp. 1-4).

Darecki, M., Edelstenne, C., Enders, T., Fernandez, E., Hartman, P., Herteman, J.-P., Kerkloh, M., King, I., Ky, P., Mathieu, M. et al. (2011). Flightpath 2050 europes vision for aviation. Off. Eur,

Déjean, B., Berthoumieu, P., \& Gajan, P. (2016). Experimental study on the influence of liquid and air boundary conditions on a planar air-blasted liquid sheet, part i: Liquid and air thicknesses. International Journal of Multiphase Flow, 79, 202-213. doi:https://doi.org/10.1016/j . ijmultiphaseflow.2015.09.002.

El-Shanawany, M., \& Lefebvre, A. (1980). Airblast atomization: effect of linear scale on mean drop size. Journal of Energy, 4, 184-189. doi:https : //doi.org/10.2514/3.62472.

Feldman, J., Tropea, C., \& Roisman, I. (2018). Measurement of liquid film thickness using laser illuminated high-speed imaging. In Symposium on the 350 Application of Laser and Imaging Techniques to Fluid Mechanics.

Friedrich, M. A., Lan, H., Wegener, J., Drallmeier, J., \& Armaly, B. F. (2008) A separation criterion with experimental validation for shear-driven films in separated flows. Journal of Fluids Engineering, 130, 051301. doi:https: //doi.org/10.1115/1.2907405.

Fu, Y., Cai, J., Jeng, S.-M., \& Mongia, H. (2005). Confinement effects on the swirling flow of a counter-rotating swirl cup. In ASME Turbo Expo 2005: Power for Land, Sea, and Air (pp. 469-478). American Society of Mechanical Engineers. doi:https ://doi .org/10.1115/GT2005-68622.

Fuster, D., Matas, J.-P., Marty, S., Popinet, S., Hoepffner, J., Cartellier, A., \& Zaleski, S. (2013). Instability regimes in the primary breakup region of planar coflowing sheets. Journal of Fluid Mechanics, 736, 150-176. doi:https ://doi.org/10.1017/jfm.2013.536.

Gepperth, S., Guildenbecher, D., Koch, R., \& Bauer, H.-J. (2010). Pre-filming primary atomization: Experiments and modeling. In 23rd European Conference on Liquid Atomization and Spray Systems (ILASS-Europe 2010), Brno, Czech Republic, September (pp. 6-8).

Gepperth, S., Koch, R., \& Bauer, H.-J. (2013). Analysis and comparison of primary droplet characteristics in the near field of a prefilming airblast atomizer. In ASME Turbo Expo 2013: Turbine Technical Conference and Exposition (pp. V01AT04A002-V01AT04A002). American Society of Mechanical Engineers. doi:https://doi .org/10.1115/GT2013-94033.

Gepperth, S., Müller, A., Koch, R., \& Bauer, H. (2012). Ligament and droplet characteristics in prefilming airblast atomization. In International Conference on Liquid Atomization and Spray Systems (ICLASS), Heidelberg, Germany, Sept (pp. 2-6).

Häber, T., Gebretsadik, M., Bockhorn, H., \& Zarzalis, N. (2015). The effect of total reflection in plif imaging of annular thin films. International Journal of Multiphase Flow, 76, 64-72. doi:https://doi.org/10.1016/j . ijmultiphaseflow.2015.06.009.

Hidrovo, C. H., Brau, R. R., \& Hart, D. P. (2004). Excitation nonlinearities in emission reabsorption laser-induced fluorescence techniques. Applied optics, 43, 894-913. doi:https://doi.org/10.1364/A0.43.000894.

Hidrovo, C. H., \& Hart, D. P. (2001). Emission reabsorption laser induced fluorescence (erlif) film thickness measurement. Measurement Science and Technology, 12, 467.

Jasuja, A. (1981). Airblast atomization of alternative liquid petroleum fuels under high pressure conditions. Journal of Engineering for Power, 103, 514-518. doi:https://doi.org/10.1115/1.3230751.

Lee, K., Aalburg, C., Diez, F. J., Faeth, G. M., \& Sallam, K. A. (2007). Primary breakup of turbulent round liquid jets in uniform crossflows. AIAA journal, 45, 1907-1916. doi:https://doi .org/https://doi.org/10. 2514/1.19397.

Lefebvre, A. H. (1980). Airblast atomization. Progress in Energy and Combustion Science, 6, 233-261. doi:https://doi.org/10.1016/
$0360-1285$ (80) 90017-9.

Les, C., Brend, M., Bonham, C., \& Carrotte, J. F. (2018). Quantifying ageing effects in thermochromic liquid crystal thermography as applied to transient convective heat transfer experiments. Measurement Science and Technology, 29, 115201.

Owen, I., \& Ryley, D. (1985). The flow of thin liquid films around corners. International journal of multiphase flow, 11, 51-62. doi:https: //doi.org/10.1016/0301-9322(85)90005-9.

Reddyhoff, T., Dwyer-Joyce, R., \& Harper, P. (2008). A new approach for the measurement of film thickness in liquid face seals. Tribology Transactions, 51,140-149. doi:https://doi.org/10.1080/10402000801918080.

Rizk, N., \& Lefebvre, A. (1980). The influence of liquid film thickness on airblast atomization. Journal of Engineering for Power, 102, 706-710.

Sallam, K., Aalburg, C., \& Faeth, G. (2004). Breakup of round nonturbulent liquid jets in gaseous crossflow. AIAA journal, 42, 2529-2540. doi:https : //doi.org/10.2514/1.3749.

Sattelmayer, T., \& Wittig, S. (1986). Internal flow effects in prefilming airblast atomizers: mechanisms of atomization and droplet spectra. Journal of engineering for gas turbines and power, 108, 465-472.

Shanmugadas, K., Chakravarthy, S., Chiranthan, R. N., Sekar, J., \& Krishnaswami, S. (2018). Characterization of wall filming and atomization inside a gas-turbine swirl injector. Experiments in Fluids, 59, 151. doi:https : //doi.org/10.1007/s00348-018-2606-0.

Vespermann, R. (2018). The influence of a multi-sector environment on leanburn gas turbine combustor aerodynamics. Ph.D. thesis Loughborough University Department of Aeronautical and Automotive Engineering.

Warncke, K., Gepperth, S., Sauer, B., Sadiki, A., Janicka, J., Koch, R., \& Bauer, H.-J. (2017). Experimental and numerical investigation of the primary breakup of an airblasted liquid sheet. International Journal of Multiphase Flow, 91, 208-224. doi:https://doi.org/10.1016/j . ijmultiphaseflow.2016.12.010.

Wu, P.-K., Kirkendall, K. A., Fuller, R. P., \& Nejad, A. S. (1997). Breakup processes of liquid jets in subsonic crossflows. Journal of Propulsion and power, 13, 64-73. doi:https: //doi .org/10.2514/2.5151. 